\title{
AN EXTENSION OF A FUNCTIONAL EQUATION OF POINCARÉ AND MANDELBROT*
}

\author{
QUANSHENG LIU ${ }^{\dagger}$
}

\begin{abstract}
We consider the distributional equation $Z \stackrel{d}{=} A_{1} Z_{1}+\ldots+A_{N} Z_{N}$, where $N, A_{i}, Z, Z_{i}$ are independent real random variables with $N \in \mathbb{N}$ and $A_{i} \geq 0$. Each $A_{i}$ is equally distributed as $A_{1}$ and each $Z_{i}$ as $Z$; the distribution of $Z$ is unknown. After establishing theorems about existence and uniqueness of solution, we show asymptotic properties at 0 of the distribution function $P[Z \leq x]$, and those at $\infty$ of the characteristic function $E e^{i t Z}$; such properties are also found for a random difference equation. In particular, we prove the absolute continuity of the distribution of $Z$ under simple moment conditions. As applications, new results are established for multiplicative cascades, for age-dependent branching processes and for branching random walks.
\end{abstract}

0. Introduction. Let $N$ be a random variable with law $\left\{p_{n}\right\}$ on $\mathbb{N}=\{0,1, \ldots\}$, and let $A$ be a random variable with values in $\mathbb{R}_{+}=[0, \infty)$. We consider the distributional equation

$$
Z \stackrel{d}{=} \sum_{i=1}^{N} A_{i} Z_{i}
$$

where $\stackrel{d}{=}$ means the equality in distribution, the real random variables $Z, Z_{i}, N, A, A_{i}$ are all independent to each other, each $A_{i}$ is equally distributed as $A$ and each $Z_{i}$ as $Z$; the distribution of $Z$ is unknown. By convention the empty sum is taken to be zero. In terms of the characteristic function $\phi(t)=E\left(e^{i t Z}\right)(t \in \mathbb{R})$, the equation reads

$$
\phi(t)=f(E \phi(A t))
$$

where $f(t)=\sum_{n=0}^{\infty} p_{n} t^{n}$ is the probability generating function of $N$.

The equation $(\mathrm{E})$ generalizes considerably the notion of stable laws (and semistable laws) where both $N$ and $A$ are constants. If $A$ is a constant with $A=1 / E N$ and $1<E N<\infty$, then the equation (E') reduces to the Poincaré's functional equation for Galton-Watson processes, whose study is a classical subject: see e.g. $[20,44,2,14,9]$. If $N$ is a constant $\geq 2$, it becomes the Mandelbrot's functional equation about multiplicative cascades $[39,40]$, and has been studied by many authors: see e.g. $[27,19,15,26,41,3]$; see also $[15,25,5,21,12,46,30,37,38]$ for some other (but not all) references. The general form of the equation is the basic equation in typical branching random walks (see Section 8), and can be applied to the study of Bellman-Harris processes (see Section 7). For many other applications of the equation, see e.g. [42] and [31].

Conditions for existence and uniqueness of positive solutions have been established in Liu [32]; the special case where $N$ is constant has been studied by Kahane and Peyrire [27], Durrett and Liggett [15] and Guivarc'h [19]. In Section 1 below we shall extend some of their results to solutions which are not necessarily positive. However, our main object is the study of asymptotic properties of the characteristic function

*Received September 28, 1999; accepted for publication November 10, 2001.

†LMAM, Univ. de Bretagne-Sud, Campus de Tohannic, BP573, 56017 Vannes, France (Quansheng.Liu@univ-ubs.fr), and IRMAR, Univ. de Rennes 1, Campus de Beaulieu, 35042 Rennes, France (liu@univ-rennes1.fr). 
and the absolute continuity for any solution, and the asymptotic behavior at 0 of the distribution function for any positive solution. To this end we first establish a result for a random difference equation which is interesting by its own (see Theorem 2.1). The main results are Theorems $3.2,3.4,3.5,4.1$ and 4.2 where a polynomial decay rate is shown for the characteristic function $E e^{i t Z}(|t| \rightarrow \infty)$ and for the distribution function $P(Z \leq x)(x \rightarrow 0)$. Our results show that the behavior of $Z$ differs according to $P(A=0)=p_{0}=p_{1}=0$ or not. These results extend those of Harris [20] (see also Bingham [8]), Athreya [2] and Dubuc [14] about the Galton-Watson process, and complete those of Bingham and Doney [9] (also for the Galton-Watson process). Exponential rates will be considered in Theorem 5.1. A description of the right tail probability $P(Z>x)(x \rightarrow \infty)$ will also be given at the end of Sections 4 and 5 , in order to compare it with the left tail probability $P(Z \leq x)(x \rightarrow 0)$.

Specified to limit variables of Mandelbrot's martingales, in addition to new results about the characteristic function and the absolute continuity, our results on the asymptotic behavior near 0 of the distribution function and on the existence of moments of negative orders make precise and complete those of Kahane [26], Molchan [41] and Barral [3, 4]: see Section 6; as has been shown by Molchan [41] and Barral $[3,4]$, such results are essential in the study of multi-fractal structure of the Mandelbrot's measure. Applied to age-dependent branching processes, our theorems enable us to improve an early result by the author: see Theorem 7.1. In Section 8, we shall see that our theorems can also be applied to obtain new results for limit variables in branching random walks.

In closing this section, let us point out that some results of this paper can be extended to the more general case where $\left\{N, A_{1}, A_{2}, \ldots\right\}$ is of arbitrary joint distribution [36], and let us introduce some notations. If $E$ is a set or a statement, we write $1_{E}$ for its indicator function; if $X$ is a random variable, we write $P_{X}$ for its probability law; if $f(t)$ and $g(t)$ are two (real or complex) functions defined for $t>0$ large (resp. small) enough, we write $f(t)=O(g(t)), t \rightarrow \infty$ (resp. $t \rightarrow 0$ ) to mean that for some constant $0<C<\infty$ and all $t>0$ large (resp. small) enough, $|f(t)| \leq C g(t)$, and we write $f(t)=o(g(t))$ if $\lim f(t) / g(t)=0$, and $f(t) \sim g(t)$ if $\lim f(t) / g(t)=1$.

1. Existence and Uniqueness of Solutions. Let $\mathbb{M}$ (resp. $\mathbb{M}_{+}$) be the set of all Borel probability measures on $\mathbb{R}$ (resp. $\mathbb{R}_{+}$). Put

$$
\alpha_{0}=P(A=0) \text { and } \tilde{N}=\sum_{i=1}^{N} 1_{\left\{A_{i}>0\right\}} \text {. }
$$

Then $\tilde{N}$ is the number of non-zero terms of $A_{i}, 1 \leq i \leq N$, whose probability generating function is

$$
\tilde{f}(t)=f\left(\alpha_{0}+\left(1-\alpha_{0}\right) t\right), \quad t \geq 0 .
$$

To avoid unimportant discussion, we suppose throughout the paper that

$$
P(\tilde{N}=0 \text { or } 1)<1 \text { and } P(A=0 \text { or } 1)<1,
$$

the contrary case being easy [32, Lemma 1.1]. For simplicity, we also assume

$$
f^{\prime}(1) \equiv E N<\infty \text { and } E A \log ^{+} A<\infty,
$$

although this is not always necessary. Let $\mathbb{F}$ (resp. $\mathbb{F}_{+}$) be the set of all non-trivial solutions in $\mathbb{M}$ (resp. $\mathbb{M}_{+}$). We say that a random variable $Z$ or a characteristic function $\phi$ is an element of $\mathbb{F}$ or $\mathbb{F}_{+}$if so is the corresponding probability measure. 
The following result gives necessary and sufficient conditions for $(\mathrm{E})$ to have nontrivial and non-negative solutions, and can be easily obtained from known results. As usual, we put $\log ^{+} x=\log x$ if $x \geq 1$, and $\log ^{+} x=0$ otherwise. Recall that a positive and measurable function $l(t)$ is called slowly varying at 0 (resp. $\infty$ ) if it is defined for all $t>0$ small (resp. large) enough such that as $t \rightarrow 0$ (resp. $\infty), l(\lambda t) / l(t) \rightarrow 1$ for each given $\lambda>0$.

THEOREM 1.1.

(i) $\mathbb{F}_{+} \neq \emptyset$ if and only if

$$
\left(1-\alpha_{0}\right) E N>1, E N E A^{\alpha}=1 \text { and } E A^{\alpha} \log A \leq 0 \text { for some } \alpha \in(0,1] .
$$

(ii) Assume (1.5). There is $\mu \in \mathbb{F}_{+}$with finite mean if and only if

$$
E N E A=1, E N \log ^{+} N<\infty \text { and } E A \log A<0
$$

when (1.6) holds, all $\mu \in \mathbb{F}_{+}$have finite mean, and are parametrized by their means; for any given function $l$ slowly varying at 0 , there is at most one element $\mu \in \mathbb{F}_{+}$ whose Laplace transform $\psi(t)=\int e^{-x t} \mu(d x)$ satisfies $\lim _{t \rightarrow 0+} \frac{1-\psi(t)}{t^{\alpha} l(t)}=1$.

( $i$ ii) If $\mu \in \mathbb{F}_{+}$, then the extinction probability $q=\mu\{0\}$ is the unique fixed point in $[0,1)$ of $\tilde{f}$.

Notice that the number $\alpha$ determined by (1.5) is necessarily unique, and is the least solution of the equation $E N E A^{x}=1, x>0$.

Proof of Theorem 1.1. Parts (i) and (iii) follow from Theorem 1.1 and Lemma 3.1 of Liu [32]. In part (ii), the third assertion is just Corollary 1.4(a) of Liu [32], the second assertion is a consequence of the first and the uniqueness theorem of Biggins and Kyprianou [7, Theorem 1.5], while the first comes from Theorem 1.1 of Liu [31] and the fact that

$$
E\left(\sum_{i=1}^{N} A_{i}\right) \log ^{+}\left(\sum_{i=1}^{N} A_{i}\right)<\infty \Leftrightarrow E A \log ^{+} A<\infty \text { and } E N \log ^{+} N<\infty,
$$

which can be proved as follows. Evidently,

$$
E\left(\sum_{i=1}^{N} A_{i}\right) \log ^{+}\left(\sum_{i=1}^{N} A_{i}\right)=p_{0}+p_{1} E A_{1} \log ^{+} A_{1}+\sum_{n \geq 2} p_{n} n E\left(A_{1} \log ^{+} \sum_{i=1}^{n} A_{i}\right) .
$$

Since $\log (x+y) \leq \log x+\log y$ if $x \geq 2$ and $y \geq 2$, we have $E\left(A_{1} \log ^{+} \sum_{i=1}^{n} A_{i}\right) \leq$ $E\left(A_{1} \log \max \left(2, \bar{A}_{1}\right)\right)+E A_{1} E \log \max \left(2, \sum_{i=2}^{n} \bar{A}_{i}\right)$ for each fixed $n \geq 2$; as $\left.E\left(\log \max \left(2, \sum_{i=2}^{n} A_{i}\right)\right) \leq \log E \max \left(2, \sum_{i=2}^{n} A_{i}\right)\right] \leq \log \left(2+n E A_{1}\right)$, this gives the implication " $\Leftarrow$ " in (1.7). To see the opposite implication, it suffices to remark that, by the law of large numbers, for some $n_{0} \in \mathbb{N}$ and all $n>n_{0}, P\left(\sum_{i=2}^{n} A_{i} \geq n E A / 2\right)>$ $1 / 2$, so that $E\left[\log \left(\sum_{i=2}^{n} A_{i}\right)\right] \geq \frac{1}{2} \log (n E A / 2)$.

We now give a theorem about existence and uniqueness of any non-trivial solution.

THEOREM 1.2.

(i) The following condition is sufficient for $\mathbb{F} \neq \emptyset$ :

$$
\left(1-\alpha_{0}\right) E N>1, E N E A^{\alpha}=1 \text { and } E A^{\alpha} \log A \leq 0 \text { for some } \alpha \in(0,2] .
$$


(ii) If (1.8) holds with $E A^{\alpha} \log A<0$, then for any given complex number $c$, there is at most one element $\phi \in \mathbb{F}$ with $\lim _{t \rightarrow 0+} \frac{1-\phi(t)}{t^{\alpha}}=c$;

(iii) Let $\delta>0$ and let $l:(0, \delta) \rightarrow(0, \infty)$ be a function slowly varying at 0 and bounded away from 0 and $\infty$ on each compact subset of $(0, \delta)$. If there is an element $\phi \in \mathbb{F}$ with $\lim _{t \rightarrow 0+} \frac{1-\phi(t)}{t^{\alpha} l(t)}=c$ for some real $\alpha \in(0,2]$ and some complex $c \neq 0$, and $E\left[A^{\alpha+\epsilon}\right]<\infty$ for some $\epsilon>0$, then $E N E A^{\alpha}=1$.

REMARKS. (1) As a consequence of part (ii) together with Theorem 1.1, if (1.6) holds (so that $\alpha=1$ ), then for any given number $c \in \mathbb{R}$, there is only one integrable solution $Z$ satisfying $E Z=c$; moreover almost surely (a.s.) $Z \geq 0$ if $c>0, Z \leq 0$ if $c<0$, and $Z=0$ if $c=0$; so under (1.6), to consider integrable elements of $\mathbb{F}$, it suffices to consider elements of $\mathbb{F}_{+}$. However, the conclusion does not hold any more if (1.6) fails: for example, when $A=1 / \sqrt{2}$ and $N=2$ a.s. (so $\alpha=2$ ), both the normal law $N(0,1)$ and the Dirac measure $\delta_{0}$ are solutions of $(\mathrm{E})$ with mean 0 . (2) In the proof, we shall see that in part (iii), when $l$ is constant, the condition $E\left(A^{\alpha+\epsilon}\right)<\infty$ can be relaxed to $E\left(A^{\alpha}\right)<\infty$. (3) If we replace the constraint $\alpha \in(0,2]$ by $\alpha>0$, and if instead of $\mathbb{F}$, we consider $\overline{\mathbb{F}}=\left\{\phi: \phi\right.$ satisfies (E') and $\phi(t)=\phi_{+}\left(|t|^{\alpha}\right)(\forall t)$, where $\phi_{+}$is the Laplace transform of some element $\left.\mu \in \mathbb{M}_{+}\right\}$, then all the conclusions (i), (ii) and (iii) still hold. In fact to see that (i) holds it suffices to take $\phi_{+}$to be the Laplace transform of a non-negative solution of (E) with $A_{i}$ replaced by $A_{i}^{\alpha}$; the proofs for (ii) and (iii) remain the same as in the proof of Theorem 1.2. Many results in the following sections also hold for elements of $\overline{\mathbb{F}}$, assuming only $\alpha>0$.

Proof of Theorem 1.2.

(i) We use an argument of Guivarc'h [19]. By Theorem 1.1, there is a non-negative and non-trivial random variable $Y$ satisfying

$$
Y \stackrel{d}{=} A_{1}^{\alpha} Y_{1}+\ldots+A_{N}^{\alpha} Y_{N}
$$

$\left\{Y_{k}\right\}$ being independent copies of $Y$ and independent of $\left\{N, A_{1}, A_{2}, \ldots\right\}$. Let $X, X_{k}$ be random variables with stable distribution of index $\alpha$, all the random variables $N, A_{k}$, $X, X_{k}, Y, Y_{k}$ being independent each other. We claim that $Z=X Y^{1 / \alpha}$ is then a solution of $(\mathrm{E})$. In fact, by the preceding equation, we need only to prove that

$$
X\left(A_{1}^{\alpha} Y_{1}+\ldots+A_{N}^{\alpha} Y_{N}\right)^{1 / \alpha} \stackrel{d}{=} A_{1} X_{1} Y_{1}^{1 / \alpha}+\ldots+A_{N} X_{N} Y_{N}^{1 / \alpha}
$$

Putting $U_{i}=A_{i} Y_{i}^{1 / \alpha}$, this just says $X\left(U_{1}^{\alpha}+\ldots+U_{N}^{\alpha}\right)^{1 / \alpha} \stackrel{d}{=} X_{1} U_{1}+\ldots+X_{N} U_{N}$, which holds by the property of stable laws with exponent $\alpha$.

(ii) Assume that $\phi_{k}$ are characteristic functions satisfying (E') and $\lim _{t \rightarrow 0+} \frac{1-\phi_{k}(t)}{t^{\alpha}}=c, k=1,2$. Write $\Delta \phi=\phi_{1}-\phi_{2}$. Then by the mean value theorem,

$$
|\Delta \phi(t)| \leq f^{\prime}(1) E|\Delta \phi(A t)|, \quad t>0 .
$$

Put $h(t)=\frac{|\Delta \phi(t)|}{t^{\alpha}}, t>0$, and let $B$ be a random variable with law $P_{B}(d x)=$ $(E N) x^{\alpha} P_{A}(d x)$. Then $P(B>0)=1$ and $h(t) \leq E h(B t), t>0$. By iteration, if $B_{1}, B_{2}, \ldots$ are independent copies of $B$, then

$$
h(t) \leq E h\left(B_{1} \ldots B_{n} t\right), \quad n \geq 1, t>0 .
$$

Let $t>0$ be fixed. As $E \log B=E N E A^{\alpha} \log A<0$, we have $B_{1} \ldots B_{n} \rightarrow 0$ a.s. by the law of large numbers. Since

$$
\lim _{x \rightarrow 0_{+}} h(x)=\lim _{x \rightarrow 0+}\left|\frac{1-\phi_{1}(x)}{x^{\alpha}}-\frac{1-\phi_{2}(x)}{x^{\alpha}}\right|=0,
$$


using the preceding inequality on $h(t)$ and the dominated convergence theorem, we obtain $h(t)=0$. This means $\phi_{1}(t)=\phi_{2}(t)$ for all $t>0$, and then for all $t \in \mathbb{R}$ because $\phi_{k}$ are hermitian symmetric.

(iii) By considering the distribution of $A$ conditional on $A>0$, we can assume $A>0$ a.s. (cf. the proof of Proposition 4.10). Let us extend the definition of $l$ by putting $l(t)=1$ if $t \geq \delta$. Then for some constant $C_{1}>0$ and all $t>0$, $1-\phi(t) \leq C t^{\alpha} l(t)$. By the functional equation (E'), we have

$$
\frac{1-\phi(t)}{t^{\alpha} l(t)}=\frac{E(1-\phi(A t))}{t^{\alpha} l(t)} \sum_{i=1}^{\infty} p_{i} \sum_{0 \leq k \leq i-1}(E \phi(A t))^{k} .
$$

It is evident that we can assume $\epsilon<\alpha$. Notice that by Potter's theorem [10, p.25, Theorem 1.5.6(ii)], there is a constant $C_{2}>0$ such that for all $x>0$ and $y>0$, $l(y) / l(x) \leq C_{2} \max \left\{(y / x)^{\epsilon},(x / y)^{\epsilon}\right\}$. Therefore

$$
\frac{(1-\phi(A t))}{t^{\alpha} l(t)}=\frac{(1-\phi(A t))}{(A t)^{\alpha} l(A t)} \frac{l(A t)}{l(t)} A^{\alpha} \leq C_{1} C_{2} \max \left(A^{\epsilon}, A^{-\epsilon}\right) A^{\alpha},
$$

so that by the dominated convergence theorem, $\lim _{t \rightarrow 0} \frac{E(1-\phi(A t))}{t^{\alpha} l(t)}=c E A^{\alpha}$. It follows by (1.10) that $c=c E A^{\alpha} \sum_{i=1}^{\infty} i p_{i}$, from which $1=E N E A^{\alpha}$. So the proof is finished.

2. A Theorem on a Random Difference Equation. In this section, $A, B, Z$ are real random variables which are not necessarily positive. We consider the random difference equation

$$
Z \stackrel{d}{=} A Z+B, \text { with } A, B, Z \text { independent of each other, }
$$

where the law of $Z$ is unknown. In terms of characteristic functions, the equation reads

$$
\phi_{Z}(t)=\phi_{B}(t) E\left[\phi_{Z}(A t)\right], \quad t \in R,
$$

where $\phi_{Y}(t)=E e^{i t Y}$ if $Y$ is a real random variable. It is well known that if $Z$ is a solution not concentrated at a point, then its distribution is either absolutely continuous or singularly continuous [18], but in general it is not easy to prove the absolute continuity. The following result gives a sufficient condition for the law of $Z$ to have a density function with some regularity properties. For all $k \in \mathbb{N}$, we denote by $C^{k}$ the class of functions $g: \mathbb{R} \rightarrow \mathbb{R}$ with $k$-fold continuous derivatives, $C^{0}$ being the class of continuous functions.

Theorem 2.1. Assume $\zeta:=\lim \sup _{|t| \rightarrow \infty}\left|\phi_{B}(t)\right|<1$ and let $Z$ be a solution of (2.1). Let $a>0$ be fixed. If $\zeta E|A|^{-a}<1$ (which reads $E|A|^{-a}<\infty$ when $\zeta=0$ ), then

$$
\left|\phi_{Z}(t)\right|=O\left(|t|^{-a}\right)(|t| \rightarrow \infty) \text { and } \int_{\mathbb{R}}\left|\phi_{Z}(t)\right||t|^{a-1} d t<\infty .
$$

In particular, if $\zeta E|A|^{-a}<1$ for some $a>1 / 2$, then the law of $Z$ has a square integrable density (with respect to the Lebesgue measure); if the condition holds for some $a \geq 1$, then the law of $Z$ has a density function of class $C^{[a]-1},[a]$ being the integral part of $a$. 
Proof of Theorem 2.1. Set $g(t)=\sup _{|s| \geq t}\left|\phi_{Z}(s)\right|, t>0$, and let $\epsilon>0$ be sufficiently small such that $(\zeta+\epsilon) E|A|^{-a}<1$. Choose $t_{0}>0$ large enough such that $\left|\phi_{B}(t)\right| \leq \zeta+\epsilon$ for all $|t|>t_{0}$. Then by the equation (2.2), for all $t>t_{0}$,

$$
g(t) \leq(\zeta+\epsilon) E g(|A| t)
$$

So the conclusions are immediate consequence of the following Lemma 2.2 (with $C=$ 0 ) which extends a result of Barral ([4]) and is also applicable in other contexts.

LEMMA 2.2. (i) Let $g: \mathbb{R}_{+} \rightarrow \mathbb{R}_{+}$be a bounded and measurable function satisfying

$$
g(t) \leq p E g(A t)+\frac{C}{t^{a}}, \quad t>t_{0}
$$

where $0<p<1, a>0, C \geq 0, t_{0} \geq 0$ are constants and $A$ is a positive random variable with $p E A^{-a}<1$. Then as $t \rightarrow \infty, g(t)=O\left(t^{-a}\right)$. (ii) We have moreover $\int_{0}^{\infty} t^{a-1} g(t) d t<\infty$ if the inequality (2.4) can be strengthened to

$$
g(t) \leq p E g(A t)+\frac{C}{t^{a} h(t)}, \quad t>t_{0}
$$

for some mesurable function $h:\left(t_{0}, \infty\right) \rightarrow(0, \infty)$ such that $\int_{t_{0}}^{\infty} \frac{1}{h(t) t} d t<\infty$.

Proof. Part (i) has been established in [33] and can be proved as follows. We can assume $t_{0}=0$ by taking $C$ large enough if necessary; then by induction, for all $n \geq 1$ and all $t>0$,

$$
g(t) \leq p^{n} E g\left(A_{1} \ldots A_{n} t\right)+C t^{-a}\left[1+p E A^{-a}+\ldots+\left(p E A^{-a}\right)^{n-1}\right],
$$

where $A_{i}$ are independent copies of $A$; letting $n \rightarrow \infty$ gives $g(t) \leq C t^{-a} /\left(1-p E A^{-a}\right)$. To prove part (ii), let $C_{1}>0$ be sufficiently large such that for all $t>0$,

$$
g(t) \leq p E g(A t)+\frac{C_{1}}{(1+t)^{a} h_{1}(t)}
$$

where $h_{1}(t)=1$ if $0<t \leq t_{0}$, and $h_{1}(t)=h(t)$ if $t>t_{0}$. Put $G(T)=\int_{0}^{T} t^{a-1} g(t) d t$ for $T>0$, and write $C_{2}=C_{1} \int_{0}^{\infty} t^{a-1}(1+t)^{-a} / h_{1}(t) d t$. Then $C_{2}<\infty$ and, by the preceding inequality, for all $T>0$,

$$
\left.G(T) \leq p E \int_{0}^{T} g(A t) t^{a-1} d t+C_{2}=p E\left[A^{-a}\right] E G(B T)\right]+C_{2}
$$

where $B$ is a random variable with distribution $P_{B}(d x)=x^{-a} P_{A}(d x) / E A^{-a}$. By iteration, for all $n \geq 1$ and all $T>0$,

$$
G(T) \leq\left(p E A^{-a}\right)^{n} E G\left(B_{1} \ldots B_{n} T\right)+C_{2}\left[1+p E A^{-a}+\ldots+\left(p E A^{-a}\right)^{n-1}\right],
$$

where $B_{i}$ are independent copies of $B$. Write $\|g\|_{\infty}=\sup _{t}|g(t)|$; notice that

$$
\begin{aligned}
\left(p E A^{-a}\right)^{n} E G\left(B_{1} \ldots B_{n} t\right) & =\left(p E A^{-a}\right)^{n} E \int_{0}^{B_{1} \ldots B_{n}} g(t) t^{a-1} d t \\
& \leq\|g\|_{\infty}\left(p E A^{-a}\right)^{n}\left(E B^{a}\right)^{n} T^{a} / a=\|g\|_{\infty} p^{n} T^{a} / a \rightarrow 0 \text { as } n \rightarrow \infty
\end{aligned}
$$


so letting $n \rightarrow \infty$ in (2.7) gives $G(T) \leq C_{2} /\left(1-p E A^{-a}\right)$. Since $T>0$ is arbitrary and the right side is independent of $T$, we obtain $\int_{0}^{\infty} t^{a-1} g(t) d t \leq C_{2} /\left(1-p E A^{-a}\right)$, as desired.

REMARKs. (1) It may be useful to remark that if $E A^{-a}<\infty$ but $p E\left[A^{-a}\right] \geq 1$ and $A$ is not a.s. constant, then the assertions (i) and (ii) also hold with $a$ replaced by $b:=a \log (1 / p) / \log E A^{-a}$. In fact, because $b / a \leq 1$, by Jensen's inequality we have $p E A^{-b}=p E\left[\left(A^{-a}\right)^{b / a}\right]<p\left[E A^{-a}\right]^{b / a}=1$, so applying the proved results for $b$ gives the desired assertions. If $A$ is a.s. a constant, say, $A=\delta \in(0,1)$, then the number $b$ defined above becomes $b=\log p / \log \delta$; this time we still have $g(t)=O\left(t^{-b}\right)$ if the condition in (ii) holds with $a$ replaced by $b$ and with $h$ non-decreasing (for example if the condition in (i) holds with $C=0$ ). In fact we can assume that $g$ is non-increasing by considering $\bar{g}(t)=\sup _{s \geq t} g(s)$ instead of $g(t)$ if necessary. By iteration, for all $n=1,2, \ldots, \phi(t) \leq p^{n} \phi\left(\delta^{n} t\right)+\frac{C}{t^{b}} \sum_{k=0}^{n-1} \frac{1}{h\left(\delta^{k} t\right)}$ if $\delta^{n} t>t_{0}$. Notice that the sum in the last display is bounded by $(1-\delta)^{-1} \sum_{k=0}^{n-1} \int_{\delta^{k+1} t}^{\delta^{k} t} \frac{1}{x h(x)} d x$, which is smaller than $(1-\delta)^{-1} \int_{t_{0}}^{\infty} \frac{1}{x h(x)} d x$ if $\delta^{n} t>t_{0}$, so that $g\left(\delta^{-n} 2 t_{0}\right) \leq C_{1}\left[\delta^{-n} 2 t_{0}\right]^{-b}$ for some constant $C_{1}>0$ and all $n \geq 1$; as $g$ is monotone, this gives $\phi(t)=O\left(t^{-b}\right)$ $(t \rightarrow \infty)$. (2) If a. s. $A>0$ and $B \geq 0$, then the conclusion of Theorem 2.1 also holds when the Fourier Transform $\phi_{Z}$ is replaced by the Laplace transform $\psi_{Z}(t)=E e^{-t Z}, t>0$. This can be easily seen by the proof. (3) By the remark (1) above, if $|A|$ is not constant and $\zeta^{-1} \leq E\left(|A|^{-a}\right)<\infty$, then the conclusions hold with $a$ replaced by $b:=a \log (1 / \zeta) / \log E A^{-a}$; when $|A|$ is constant, say $|A|=\delta \in(0,1)$, then $\mid \phi_{Z}(t)=O\left(|t|^{-b}\right)(|t| \rightarrow \infty)$, where $b=\log \zeta / \log \delta$. This remark have evident consequences in the following two sections, but for simplicity we shall not mention them.

Let us give some comments for the case where $A=\delta$ is a constant in $(0,1)$. In this case a solution of (2.1) can be expressed as the sum of a random Taylor series:

$$
Z=\sum_{n=0}^{\infty} B_{n} \delta^{n}
$$

where $\left\{B_{n}\right\}$ is a sequence of independent and identically distributed (i.i.d.) real random variables; by the formula of the radius of convergence of a Taylor series, the series converges a.s. for each $\delta \in(0,1)$ if $E \log ^{+}\left|B_{0}\right|<\infty$ (which implies that a.s. $\lim \sup _{n \rightarrow \infty} \frac{\log ^{+}\left|B_{n}\right|}{n}=0$ by the law of large numbers).

Assume $\delta \in(0,1)$ and $E \log ^{+}\left|B_{0}\right|<\infty$, and let $Z$ be defined by (2.8). An interesting question is to study asymptotic properties of the characteristic function of $Z$, and to know when its distribution is absolutely continuous. By the second remark after the proof of Theorem 2.1, we know that if

$$
\zeta:=\limsup _{|t| \rightarrow \infty}\left|E e^{i t B_{0}}\right|<1,
$$

then as $|t| \rightarrow \infty,\left|E e^{i t Z}\right|=O\left(|t|^{-b}\right)$, where $b=\log \zeta / \log \delta$, so that the distribution of $Z$ has a square integrable density if $\zeta^{2}<\delta$. Of particular interest is the case where the law of $B_{0}$ is a Cantor-like measure, for which K.S.Lau and T.Y.Hu [23] have recently obtained some interesting results about the value of $\zeta$. When $\left\{B_{n}\right\}$ is a Rademacher sequence, the study of the series (2.8) is then a classical subject: see for example $[16],[24],[22]$ and [45]. However in this case our result gives no information (because $\zeta=1)$. 
3. Asymptotic Properties of $\phi \in \mathbb{F}$ and Absolute Continuity . In this section, for simplicity we assume $\alpha_{0}=p_{0}=0$; this condition may be removed by an argument of reduction (cf. Proposition 4.10). Recall that we always assume (1.3), (1.4), and that $\mathbb{F}$ (resp. $\mathbb{F}_{+}$) stands for the set of all non-trivial solutions (resp. non-negative solutions) of (E).

For positive solutions with finite mean, the following result is a consequence of a theorem of Biggins and Grey [6]. The basic idea in the following proof is inspired by an argument of Athreya [1]; some similar arguments were used in [6].

Lemma 3.1. Assume (1.8) and $\alpha_{0}=p_{0}=0$. Let $\mu \in \mathbb{F}$ and let $\phi$ be its characteristic function. Then $\lim _{|t| \rightarrow \infty} \phi(t)=0$.

Proof. Set $l=\lim \sup _{t \rightarrow \infty}|\phi(t)|$. Since $\phi$ is Hermitian symmetric, it suffices to prove that $l=0$.

(i) We first prove that $l=0$ or 1 . By (E'), $|\phi(t)| \leq f(E|\phi(A t)|), t \in \mathbb{R}$. Letting $t \rightarrow \infty$ and using Fatou's lemma, we obtain $l \leq f(l)$. Therefore $l=0$ or 1 , noting that $f(x)<x$ if $0<x<1$.

(ii) We next prove that for all $t \neq 0,|\phi(t)|<1$. Otherwise, by Lemma 4 of Chap.IV.1 of Feller [17], there is some $h>0$ such that $|\phi(h)|=1$ and $|\phi(t)|<1$ if $0<t<h$. So $1=|\phi(h)|=f(E \phi(A h)) \leq E \phi(A h) \mid$. Therefore, a.s. $|\phi(A h)|=1$. Since $P(0<A<1)>0$, it follows that for some $0<a<1,|\phi(a h)|=1$, which is a contradiction with the definition of $h$.

(iii) We then show that $l<1$. Assume $l=1$. Let $0<t_{0}<\infty$ be arbitrary fixed, and let $0<\epsilon<1-\left|\phi\left(t_{0}\right)\right|$. Choose $t_{1}=t_{1}(\epsilon)$ and $t_{2}=t_{2}(\epsilon)$ such that $0<t_{1}<t_{0}<t_{2}<\infty$, with

$$
\left|\phi\left(t_{1}\right)\right|=\left|\phi\left(t_{2}\right)\right|=1-\epsilon \text { and }|\phi(t)| \leq 1-\epsilon \text { if } t \in\left[t_{1}, t_{2}\right] .
$$

This is possible since $\phi$ is continuous and $|\phi(0)|=\limsup _{t \rightarrow \infty}|\phi(t)|=1$. By the equation (E'), for all $t \in \mathbb{R},|\phi(t)| \leq E|\phi(A t)|$. It follows that if $A_{i}(i \geq 1)$ are independent copies of $A$, then: $|\phi(t)| \leq E\left|\phi\left(A_{1} \ldots A_{n} t\right)\right|, E|\phi(A t)| \leq E\left|\phi\left(A_{1} \ldots A_{n} t\right)\right|$, and

$$
|\phi(t)| \leq f(E|\phi(A t)|) \leq f\left(E\left|\phi\left(A_{1} \ldots A_{n} t\right)\right|\right) \quad(n \geq 1, t \in \mathbb{R}) .
$$

Therefore, using $\left|\phi\left(A_{1} \ldots A_{n} t_{2}\right)\right| \leq 1-\epsilon$ if $t_{1}<A_{1} \ldots A_{n} t_{2} \leq t_{2}$ and $\left|\phi\left(A_{1} \ldots A_{n} t_{2}\right)\right| \leq 1$ otherwise, we obtain:

$$
\begin{gathered}
\begin{array}{c}
E\left|\phi\left(A_{1} \ldots A_{n} t_{2}\right)\right| \leq(1-\epsilon) P\left[t_{1}<A_{1} \ldots A_{n} t_{2} \leq t_{2}\right]+1-P\left[t_{1}<A_{1} \ldots A_{n} t_{2} \leq t_{2}\right] \\
=1-\epsilon P\left[t_{1}<A_{1} \ldots A_{n} t_{2} \leq t_{2}\right]
\end{array} \\
1-\epsilon=\left|\phi\left(t_{2}\right)\right| \leq f\left(E\left|\phi\left(A_{1} \ldots A_{n} t_{2}\right)\right|\right) \leq f\left(1-\epsilon P\left[t_{1}<A_{1} \ldots A_{n} t_{2} \leq t_{2}\right]\right), \\
\frac{1-f\left(1-\epsilon P\left[t_{1}<A_{1} \ldots A_{n} t_{2} \leq t_{2}\right]\right)}{\epsilon P\left[t_{1}<A_{1} \ldots A_{n} t_{2} \leq t_{2}\right]} \leq \frac{1}{P\left[t_{1}<A_{1} \ldots A_{n} t_{2} \leq t_{2}\right]} .
\end{gathered}
$$

Since $\lim _{\epsilon \rightarrow 0} t_{1}(\epsilon)=0$ (this can be easily verified) and $t_{1}(\epsilon) / t_{2}(\epsilon) \leq t_{1}(\epsilon) \rightarrow 0(\epsilon \rightarrow 0)$, letting $\epsilon \rightarrow 0$ in the above inequality gives

$$
f^{\prime}(1) \leq 1 / P\left[A_{1} \ldots A_{n} \leq 1\right]
$$


Let $\alpha \in(0,2]$ be defined as in (1.8), so that $E A^{\alpha}=1 / E N<1$. Then by Markov's inequality,

$$
P\left[A_{1} \ldots A_{n}>1\right]=P\left[\left(A_{1} \ldots A_{n}\right)^{\alpha}>1\right] \leq\left[E\left(A^{\alpha}\right)\right]^{n} \rightarrow 0 \quad \text { as } n \rightarrow \infty .
$$

So $\lim _{n \rightarrow \infty} P\left[A_{1} \ldots A_{n} \leq 1\right]=1$. Therefore, letting $n \rightarrow \infty$ in the preceding inequality on $f^{\prime}(1)$ gives $f^{\prime}(1) \leq 1$. This contradicts the hypothesis that $f^{\prime}(1)=E N>1$. So $l<1$.

ThEorem 3.2. Assume (1.8) and $\alpha_{0}=p_{0}=0$. Let $\mu \in \mathbb{F}$ and let $\phi$ be its characteristic function. Write $m=\min \left\{i \geq 1: p_{i}>0\right\}$ and let $0<a<\infty$ be a positive number.

(i) If $p_{1} E A^{-a}<1$ (which reads $E A^{-a}<\infty$ when $p_{1}=0$ ), then

$$
\phi(t)=O\left(|t|^{-m a}\right)(|t| \rightarrow \infty) \text { and } \int_{-\infty}^{\infty}|\phi(t)||t|^{m a-1} d t<\infty .
$$

(ii) When $p_{1}=0$, the condition $P(A \leq x)=O\left(x^{-a}\right)(x \rightarrow 0)$ suffices for $\phi(t)=$ $O\left(|t|^{-m a}\right)(|t| \rightarrow \infty)$.

Proof. (i) We notice that the equation (E) can be regarded as a special case of the random difference equation (2.1) with $B=\sum_{2 \leq i \leq N} A_{i} Z_{i}$ (the empty sum is taken to be 0 ), whose characteristic function is $\phi_{B}(t)=f_{1}(E \phi(A t)), f_{1}$ being the probability generating function of $N-1$. By Lemma $3.1, \zeta:=\lim _{|t| \rightarrow \infty}\left|\phi_{B}(t)\right|=p_{1}$; so by Theorem 2.1, $p_{1} E A^{-a}<1$ implies

$$
\phi(t)=O\left(|t|^{-a}\right)(|t| \rightarrow \infty) \text { and } \int_{-\infty}^{\infty}|\phi(t)||t|^{a-1} d t<\infty .
$$

This ends the proof of (3.1) in the case where $p_{1}>0$. Assume $p_{1}=0$. Then $m \geq 2$ and by the equation (E'),

$$
|\phi(t)| \leq f(E|\phi(A t)|) \leq(E|\phi(A t)|)^{m} .
$$

By (3.2), there is a constant $C>0$ such that $|\phi(t)| \leq C|t|^{-a}$ for all $t \neq 0$, so that $E|\phi(A t)| \leq E|C A t|^{-a}=C E A^{-a}|t|^{-a}$. Consequently by (3.3), $|\phi(t)| \leq$ $C^{m}\left(E A^{-a}\right)^{m}|t|^{-m a}$ and

$$
\begin{aligned}
\int_{-\infty}^{\infty}|\phi(t)||t|^{m a-1} d t & \leq \int_{-\infty}^{\infty}\left(E|\phi(A t)||t|^{a}\right)^{m-1} E|\phi(A t)||t|^{a-1} d t \\
& \leq\left(C E A^{-a}\right)^{m-1} E \int_{-\infty}^{\infty}|\phi(A t)||t|^{a-1} d t \\
& =\left(C E A^{-a}\right)^{m-1} E A^{-a} \int_{-\infty}^{\infty}|\phi(x)||x|^{a-1} d x
\end{aligned}
$$

The last integral is finite by (3.2). So the proof of (i) is finished.

(ii) Under the given conditions, we have $m \geq 2$ and $E A^{-a_{1}}<\infty$ for all $0<a_{1}<a$, so the conclusion in part (i) implies $\phi(t)=O\left(|t|^{-b}\right)$ for for all $0<b<m a$. Now choose $b \in(a, m a)$ and let $C_{1}$ be such that $|\phi(t)| \leq C_{1}(1+|t|)^{-b}$ for all $t \neq 0$. Then a similar argument as above gives

$$
\phi(t) \leq[E \phi(A t)]^{m} \leq C_{1}^{m}\left(E\left[(1+A|t|)^{-b}\right]\right)^{m}=O\left(|t|^{-m a}\right),
$$


where the last step holds by the following Lemma.

Lemma 3.3. [33, Lemma 4.3] If $P(A \leq x)=O\left(x^{a}\right)(x \rightarrow 0)$ for some $a>0$, then for all $b>a$,

$$
E\left[(1+A t)^{-b}\right]=O\left(t^{-a}\right) \text { as } t \rightarrow \infty .
$$

When $\mu \in \mathbb{F}_{+}$has finite mean, a similar argument as in the proof of Theorem 3.2 gives the decay rate of the derivative $\phi^{\prime}(t), \phi$ being the characteristic function of $\mu$ :

Theorem 3.4. Assume (1.6) and $\alpha_{0}=p_{0}=0$. Let $\mu \in \mathbb{F}_{+}$and let $\phi$ be its characteristic function. Write $m=\inf \left\{i \geq 1: p_{i}>0\right\}$ and let $0 \leq a<\infty$ be a non-negative number.

(i) If $p_{1} E A^{-a}<1$ (which reads $E A^{-a}<\infty$ when $p_{1}=0$ ), then

$$
\phi^{\prime}(t)=O\left(|t|^{-(1+m a)}\right)(|t| \rightarrow \infty) \text { and } \int_{-\infty}^{\infty}\left|\phi^{\prime}(t)\right||t|^{m a} d t<\infty .
$$

(ii) When $p_{1}=0$, the condition $P(A \leq x)=O\left(x^{-a}\right)(x \rightarrow 0)$ suffices for $\phi^{\prime}(t)=O\left(|t|^{-(1+m a)}\right)(|t| \rightarrow \infty)$.

Proof. Without loss of generality, we assume $\int x \mu(d x)=1$. Still by the equation (E'), we have

$$
\phi^{\prime}(t)=f^{\prime}(E \phi(A t)) E \phi^{\prime}(A t) A, \quad t \in \mathbb{R} .
$$

Let $\tilde{Z}$ and $\tilde{A}$ be random variables with distributions $x \mu(d x)$ and $f^{\prime}(1) x P_{A}(d x)$ respectively, and let $\tilde{B}$ be a random variable with characteristic function $\phi_{\tilde{B}}(t)=$ $f^{\prime}(E \phi(A t)) / f^{\prime}(1) ; \tilde{Z}, \tilde{A}$ and $\tilde{B}$ being independent of each other. Then $\tilde{Z}$ has characteristic function $\phi_{\tilde{Z}}(t)=\phi^{\prime}(t) / i$, and the preceding identity reads

$$
\phi_{\tilde{Z}}(t)=\phi_{\tilde{B}}(t) E \phi_{\tilde{Z}}(\tilde{A} t), \quad t \in \mathbb{R} \text {. }
$$

(i) Notice that $\tilde{\zeta}:=\lim _{|t| \rightarrow \infty}\left|\phi_{\tilde{B}}(t)\right|=f^{\prime}(0) / f^{\prime}(1)=p_{1} / f^{\prime}(1)$ and $\tilde{\zeta} E|\tilde{A}|^{-(a+1)}=$ $p_{1} E A^{-a}<1$, so by Theorem 2.1 ,

$$
\phi^{\prime}(t)=O\left(|t|^{-(1+a)}\right)(|t| \rightarrow \infty) \text { and } \int_{-\infty}^{\infty}\left|\phi^{\prime}(t)\right||t|^{a} d t<\infty .
$$

This completes the proof of (3.5) in the case where $p_{1}>0$. Assume $p_{1}=0$. Then $m \geq 2$. Because $\left|\phi_{\tilde{B}}(t)\right| \leq(E|\phi(A t)|)^{m-1}$, it follows that for all $t \in \mathbb{R}$,

$$
\left|\phi_{\tilde{Z}}(t)\right| \leq(E|\phi(A t)|)^{m-1} E\left[\left|\phi_{\tilde{Z}}(\tilde{A} t)\right|\right] .
$$

Recall that $|\phi(t)|=O\left(|t|^{-m a}\right)$ by Theorem 3.2 and $\phi^{\prime}(t)=O\left(|t|^{-(1+a)}\right)$ by (3.8); so for some constant $C>0$ and all $t \in \mathbb{R},|\phi(t)| \leq C|t|^{-a}$ and $\left|\phi_{\tilde{Z}}\right|=\left|\phi^{\prime}(t)\right| \leq C|t|^{-(1+a)}$. Therefore by (3.9),

$$
\left|\phi_{\tilde{Z}}(t)\right| \leq\left(E C|A t|^{-a}\right)^{m-1} E\left[C|\tilde{A} t|^{-(1+a)}\right]=C_{1}|t|^{-(m a+1)}
$$

where $C_{1}=C^{m}\left(E A^{-a}\right)^{m-1} E\left[\tilde{A}^{-(1+a)}\right]=f^{\prime}(1) C^{m}\left(E A^{-a}\right)^{m}<\infty$. This gives the first assertion in (3.5). For the second assertion, we remark that for all $t$, $\left(E|\phi(A t)| t^{a}\right)^{m-1} \leq\left(C E A^{-a}\right)^{m-1}=: C_{2}$, so that by (3.9),

$$
\begin{aligned}
\int_{-\infty}^{\infty}\left|\phi_{\tilde{Z}}(t)\right||t|^{m a} d t & \leq C_{2} E \int_{-\infty}^{\infty}\left|\phi_{\tilde{Z}}(\tilde{A} t)\right||t|^{a} d t \\
& =C_{2} E \tilde{A}^{-(a+1)} \int_{-\infty}^{\infty}\left|\phi_{\tilde{Z}}(x)\right||x|^{a} d x
\end{aligned}
$$


Because $E \tilde{A}^{-(a+1)}=f^{\prime}(1) E A^{-a}<\infty$, the second assertion in (3.5) follows from (3.11) and (3.8).

(ii) Under the given conditions, we have $m \geq 2$ and $E A^{-a_{1}}<\infty$ for all $a_{1} \in(0, a)$, so by the conclusion in part (i), we know that $\phi_{\bar{Z}}(t)=O\left(|t|^{-(1+m b)}\right)(|t| \rightarrow \infty)$ for all $0<b<a$. Choose $b \in(a / m, a)$ and let $C_{3}>0$ be a constant such that for all $t \in \mathbb{R}$, $|\phi(t)| \leq C_{3}|t|^{-a}$ and $\left|\phi^{\prime}(t)\right| \leq C_{3}(1+|t|)^{-(1+m b)}$. Then for all $t \in \mathbb{R}$,

$$
\left|\phi_{\tilde{Z}}(t)\right| \leq(E|\phi(A t)|)^{m-1} E\left[\left|\phi_{\tilde{Z}}(\tilde{A} t)\right|\right] \leq\left(E C_{3}|A t|^{-a}\right)^{m-1} E\left[C_{3}(1+|\tilde{A} t|)^{-(1+m b)}\right]
$$

Since $P(\tilde{A} \leq x)=O\left(x^{1+a}\right)(x \rightarrow 0)$ and $1+m b>1+a$, we have $E\left[(1+|\tilde{A} t|)^{-(1+m b)}\right]=$ $O\left(|t|^{-(1+a)}\right)$ by Lemma 3.3 ; it follows by the preceding inequality on $\phi_{\bar{Z}}(t)$ that $\left|\phi_{\bar{Z}}(t)\right|=O\left(|t|^{-(m a+1)}\right)$.

Notice that the condition $p_{1} E A^{-a}<1$ always holds for $a=0$, so that by Theorem 3.4(i), under (1.6), all $\phi \in \mathbb{F}_{+}$satisfies

$$
\phi^{\prime}(t)=O\left(|t|^{-1}\right)(|t| \rightarrow \infty) \text { and } \int_{-\infty}^{\infty}\left|\phi^{\prime}(t)\right| d t<\infty .
$$

As an application of Theorems 3.2 and 3.4, we obtain the absolute continuity of $\mu \in \mathbb{F}$ under simple moment conditions:

TheOREM 3.5. Assume (1.8) and $\alpha_{0}=p_{0}=0$. Let $\mu \in \mathbb{F}$ and let $\phi$ be its characteristic function. Write $m=\min \left\{i \geq 1: p_{i}>0\right\}$. Then $\mu$ has a density function with respect to the Lebesgue measure if one of the following conditions holds ${ }^{1}$ : (a) $p_{1}=0$ and $E A^{-a}<\infty$ for some $a>1 /(2 m)$; (b) $p_{1}>0$ and $p_{1} E A^{-a}<1$ for some $a>1 / 2$; (c) (1.6) and $\mu \in \mathbb{F}_{+} ;$(d) $\mu \in \mathbb{F}_{+}, \alpha<1, A$ is not a.s. concentrated on a geometric series, and $E N^{1+\epsilon}+E A^{1+\epsilon}<\infty$ for some $\epsilon>0$. Moreover, if (a) or (b) holds with $a \geq 1 / m$, then $\mu$ has a density function of class $C^{[m a]-1}$, given by

$$
x \mapsto \frac{1}{2 \pi} \int_{-\infty}^{\infty} \phi(t) e^{-i t x} d t ;
$$

when (c) holds, $\mu$ has always a continuous density function on $(0, \infty)$, given by

$$
x \mapsto \frac{1}{2 \pi i x} \int_{-\infty}^{\infty} \phi^{\prime}(t) e^{-i t x} d t
$$

which is of class $C^{[m a]}$ if additionally (a) or (b) holds with $a \geq 1 / m$.

Proof. If (a) or (b) holds, then $\phi$ is square integrable by the first assertion in part (i) of Theorem 3.2, so that $\mu$ has a square integrable density function; if the condition holds with $a \geq 1 / m$, then by the second assertion in part (i) of the same theorem, $\phi$ is integrable and $\mu$ has a density function of class $C^{[m a]-1}$ given by (3.13). If (c) holds, then the measure $x \mu(d x)$ has Fourier transform $t \mapsto \phi^{\prime}(t) / i$, which is always integrable by Theorem 3.4 (i) (cf. (3.12)), so that the measure $x \mu(d x)$ has a density function given by $x \mapsto \frac{1}{2 \pi i} \int_{-\infty}^{\infty} \phi^{\prime}(t) e^{-i t x} d t$; this density function is of class $C^{[m a]}$ again by Theorem 3.4 (i). If (d) holds, then $\mu$ is absolutely continuous because, by [32, Theorem 7.3], $\mu$ is the distribution of $X Y^{1 / \alpha}$, where $Y$ is a non-negative and non-trivial solution of (1.9), $X$ is of stable law with index $\alpha$ and independent of $Y$.

We remark that in Case (c), Theorem 3.4 gives more information about the existence of a density and its regularity than what we can obtain from Theorem 3.2.

\footnotetext{
${ }^{1}$ Since the completion of this paper, the author has recently proved in [36] (and in a more general setting) that $\mu$ has a density function on $(0, \infty)$ whenever $E A^{-\epsilon} 1\{A>0\}<\infty$ for some $\epsilon>0$.
} 
4. Polynomial Tail Behavior of $\mu \in \mathbb{F}_{+}$. In this section, we consider the case where $P(0<Z \leq x)$ decays at a polynomial rate as $x \rightarrow 0+$. The main results of this section are the following two theorems. We distinguish two cases according as $\alpha_{0}=p_{0}=p_{1}=0$ or not. Recall again that we always assume (1.3) and (1.4).

Theorem 4.1. Assume (1.5) and $\alpha_{0}=p_{0}=p_{1}=0$, and let $Z$ be a non-trivial and non-negative solution of $(E)$. Write $m=\min \left\{i \geq 2: p_{i}>0\right\}$. Then for each fixed $0<a<\infty$, the following assertions hold:

(i) As $x \rightarrow 0, P(A \leq x)=O\left(x^{a}\right)$ if and only if $P(Z \leq x)=O\left(x^{m a}\right)$.

(ii) If for some constants $C_{1}>0, C_{2}>0$ and all $x>0$ small enough, $C_{1} x^{a} \leq$ $P(A \leq x) \leq C_{2} x^{a}$, then there are some constants $C_{3}>0, C_{4}>0$ such that, for all $x>0$ small enough,

$$
C_{3} x^{m a} \leq P(Z \leq x) \leq C_{4} x^{m a} .
$$

(iii) If $E\left(A^{-a}\right)<\infty$, then $E\left[Z^{-m a}\right]<\infty$; conversely, if $E\left[Z^{-m a}\right]<\infty$, then $E\left(A^{-b}\right)<\infty$ for all $0<b<a$.

(iv) Let $\delta>0$ and let $l:(0, \delta] \rightarrow(0, \infty)$ be a function slowly varying at 0 and bounded away from 0 and $\infty$ on each compact subset of $(0, \delta]$. If $P(A \leq x) \sim$ $x^{a} l(x)(x \rightarrow 0)$, then

$$
P(Z \leq x) \sim p_{m} x^{m a}\left[E\left(Z^{-a}\right)\right]^{m}[l(x)]^{m}(x \rightarrow 0) .
$$

REMARK. The converse in part (iii) cannot be improved to " $E\left[Z^{-m a}\right]<\infty \Rightarrow$ $E\left(A^{-a}\right)<\infty$." To see this, let $A$ be such that for some $a>0, b \in \mathbb{R}$ and $C>0$, $P(A \leq x) \sim C x^{a}|\log x|^{-b}(x \rightarrow 0)$. Then by part (iv) of Theorem 4.1, $P(Z \leq x) \sim$ $C^{m} x^{m a} E^{m}\left(Z^{-a}\right)|\log x|^{-m b}(x \rightarrow 0)$. So for some constants $C_{1}>0$ and $C_{2}>0$, $E\left(Z^{-m a}\right)=m a \int_{0}^{\infty} P(Z \leq 1 / y) y^{m a-1} d y \leq C_{1}+C_{2} \int_{2}^{\infty} y^{-m a}|\log y|^{-m b} y^{m a-1} d y=$ $C_{1}+C_{2} \int_{2}^{\infty} \frac{d y}{y(\log y)^{m b}}$, which is finite if $m b>1$. Similarly, for some constant $C_{3}>0$, $E\left(A^{-a}\right)=a \int_{0}^{\infty} P(A \leq 1 / y) y^{a-1} d y \geq C_{3} \int_{2}^{\infty} \frac{d y}{y(\log y)^{b}}=\infty$ if $b \leq 1$. So for $1 / m<$ $b \leq 1, E\left(Z^{-m a}\right)<\infty$ but $E\left(A^{-a}\right)=\infty$.

THEOREM 4.2. Assume (1.5) and $\alpha_{0}+p_{0}+p_{1}>0$, and let $Z$ be a non-trivial and non-negative solution of $(E)$. Write $\tilde{p}_{1}=\left(1-\alpha_{0}\right) f^{\prime}\left(\alpha_{0}+\left(1-\alpha_{0}\right) q\right)$. Then $0<\tilde{p}_{1}<1$, and for each fixed $0<a<\infty$, the following assertions hold:

(i) If $\tilde{p}_{1} E\left[A^{-a} \mid A>0\right]<1$, then $E\left[Z^{-b} \mid Z>0\right]<\infty$ for all $0<b<a$; conversely, if $E\left[Z^{-a} \mid Z>0\right]<\infty$, then $\tilde{p}_{1} E\left[A^{-a} \mid A>0\right]<1$;

(ii) In the case where $A \leq 1$ almost surely, $\tilde{p}_{1} E\left[A^{-a} \mid A>0\right]<1$ if and only if $E\left[Z^{-a} \mid Z>0\right]<\infty$.

Our result gives explicitly the critical value for existence of moments of negative orders:

Corollary. Under the assumptions of Theorem 4.2, If $\tilde{p}_{1} E\left[A^{-\lambda} \mid A>0\right]=1$ for some $\lambda>0$, then

$$
E\left[Z^{-b} \mid Z>0\right] \begin{cases}<\infty & \text { when } b \in(0, \lambda), \text { and } \\ =\infty & \text { when } b \in[\lambda, \infty) .\end{cases}
$$

Theorems 4.1 and 4.2 show that there are two essentially different cases, according to $\alpha_{0}=p_{0}=p_{1}=0$ or not. For the Mandelbrot's equation, the fact that the situation differs according to $P(A=0)=0$ or not has already been remarked by Barral [3, 4], 
although his description was not so precise as is given by our results above. For the Poincaré's equation, the results show that $Z$ has moments of all negative orders if $p_{0}=p_{1}=0$, and that there is an critical value for existence of negative orders if $p_{0}+p_{1}>0$; in fact it is known [8] that as $x \rightarrow 0, P(0<Z \leq x)$ decays at an exponential rate if $p_{0}=p_{1}=0$ (see also Theorem 5.1 in Section 5), and at a polynomial rate if $p_{0}+p_{1}>0$.

We proceed towards the proof of Theorems 4.1 and 4.2 by a series of lemmas and propositions. In the following, we always assume (1.5) and let $Z$ be a non-trivial and non-negative solution of $(\mathrm{E})$.

LEMMA 4.3. [33] Let $X>0$ be a positive random variable. For each fixed $0<a<\infty$, consider the following statements: (i) $E\left[X^{-a}\right]<\infty$; (ii) $E\left[e^{-t X}\right]=$ $O\left(t^{-a}\right)(t \rightarrow \infty)$; (iii) $P[X \leq x]=O\left(x^{a}\right)(x \rightarrow 0)$; (iv) $\forall b \in(0, a), E\left[X^{-b}\right]<\infty$. Then the following implications hold: $(i) \Rightarrow(i i) \Leftrightarrow(i i i) \Rightarrow(i v)$.

Proposition 4.4. Assume $\alpha_{0}=p_{0}=0$ and write $m=\min \left\{i \geq 1: p_{i}>0\right\}$. Let $0<a<\infty$ be a positive number. (i) If $E e^{-Z t}=O\left(t^{-a}\right)(t \rightarrow \infty)$, then $E e^{-A t}=O\left(t^{-a / m}\right)(t \rightarrow \infty)$. (ii) If $E Z^{-a}<\infty$, then $E A^{-a}<\infty$ when $m=1$, and $E A^{-b / m}<\infty$ when $b \in(0, a)$ and $m>1$.

Proof. Write $\phi_{A}(t)=E\left[e^{-A t}\right]$. Let $0<c<\infty$ be such that $P(Z \leq c)>0$. Then by $(\mathrm{E})$, for all $t>0$,

$$
\begin{aligned}
E\left[e^{-t Z}\right] & =E\left[e^{-t \sum_{i=1}^{N} A_{i} Z_{i}}\right] \\
& =\sum_{i=m}^{\infty} p_{i}\left[E \phi_{A}(Z t)\right]^{i} \geq p_{m}\left[E \phi_{A}(Z t)\right]^{m} \geq p_{m}\left[P(Z \leq c) \phi_{A}(c t)\right]^{m} .
\end{aligned}
$$

Therefore $E\left[e^{-t Z}\right]=O\left(t^{-a}\right)$ implies $\phi_{A}(t)=O\left(t^{-a / m}\right)(t \rightarrow \infty)$, which ends the proof of (i). To prove (ii), we assume $E Z^{-a}<\infty$ and write $Z^{\prime}=A_{1} Z_{1}+\ldots+A_{N} Z_{N}$. Then we have consecutively,

$$
\begin{aligned}
Z^{\prime} & \leq \max \left\{A_{1}, \ldots, A_{N}\right\}\left(Z_{1}+\ldots+Z_{N}\right), \\
\left(Z^{\prime}\right)^{-a} & \geq\left(\max \left\{A_{1}, \ldots, A_{N}\right\}\right)^{-a}\left(Z_{1}+\ldots+Z_{N}\right)^{-a}, \\
E Z^{-a} & =E\left(Z^{\prime}\right)^{-a} \geq E\left[\left(\max \left\{A_{1}, \ldots, A_{N}\right\}\right)^{-a}\left(Z_{1}+\ldots+Z_{N}\right)^{-a} 1\{N=m\}\right] \\
& =E\left[\max \left\{A_{1}, \ldots, A_{m}\right\}\right]^{-a} E\left(Z_{1}+\ldots+Z_{m}\right)^{-a} p_{m} .
\end{aligned}
$$

Therefore $E\left(\max \left\{A_{1}, \ldots, A_{m}\right\}\right)^{-a}<\infty$. If $m=1$, this just says $E A^{-a}<\infty$; if $m>1$, then as $x \rightarrow 0, P\left[\max \left\{A_{1}, \ldots, A_{m}\right\} \leq x\right]=O\left(x^{a}\right)$, so that $P[A \leq x]=O\left(x^{a / m}\right)$ $(x \rightarrow 0)$ and $E A^{-b / m}<\infty$ for all $0<b<a$ by Lemma 4.3.

Proposition 4.5. Assume $\alpha_{0}=p_{0}=p_{1}=0$ and write $m=\min \left\{i \geq 1: p_{i}>0\right\}$. Let $0<a<\infty$ be a positive number. If either (i) $p_{1}=0$ and $P(A \leq x)=O\left(x^{a}\right)$ as $x \rightarrow 0$, or (ii) $p_{1}>0$ and $p_{1} E A^{-a}<1$, then $E e^{-Z t}=O\left(t^{-m a}\right)$ as $t \rightarrow \infty$.

Proof. The same as in the proof of Theorem 4.2, using the Laplace transform instead of the characteristic function, together with the remark (2) following the proof of Theorem 2.1.

LEMma 4.6. Assume $p_{0}=0$ and put $m=\min \left\{k \geq 1: p_{k}>0\right\}$. Then for all fixed $K>0$ and all $x>0, P(Z \leq x) \geq p_{m}[P(Z \leq K)]^{m}\left[P\left(A \leq \frac{x}{K m}\right)\right]^{m}$.

Proof. It suffices to use (E) and the fact that

$P\left(A_{1} Z_{1}+\ldots+A_{N} Z_{N} \leq x\right) \geq P\left(N=m, Z_{i} \leq K\right.$ and $A_{i} K \leq x / m$ for all $\left.i=1, \ldots, m\right)$. 
LEMMA 4.7. Let $n \geq 2$ be an integer and let $X_{1}, \ldots, X_{n}$ be a sequence of i.i.d. positive random variables. Then for all $a>0, E\left[\left(X_{1}+\ldots+X_{n}\right)^{-n a}\right] \leq n\left[E\left(X_{1}^{-a}\right)\right]^{n}$. Then

Proof. If $E\left(X_{1}^{-a}\right)=\infty$, there is nothing to prove. Assume that $E\left(X_{1}^{-a}\right)<\infty$.

$$
E\left(X_{1}^{-a}\right)=\int_{0}^{\infty} P\left(X_{1}^{-a}>x\right) d x=a \int_{0}^{\infty} P\left(X_{1}<1 / y\right) y^{a-1} d y .
$$

Similarly, $E\left[\left(X_{1}+\ldots+X_{n}\right)^{-n a}\right]=n a \int_{0}^{\infty} P\left(X_{1}+\ldots+X_{n}<1 / y\right) y^{n a-1} d y \leq$ na $\int_{0}^{\infty}\left[P\left(X_{1}<1 / y\right)\right]^{n} y^{n a-1} d y$. Since $P\left(X_{1}<1 / y\right)=P\left(X_{1}^{-a}>y^{a}\right) \leq y^{-a} E\left(X_{1}^{-a}\right)$, it follows that

$$
\begin{aligned}
E\left[\left(X_{1}+\ldots+X_{n}\right)^{-n a}\right] & \leq n a \int_{0}^{\infty}\left[y^{-a} E\left(X_{1}^{-a}\right)\right]^{n-1}\left[P\left(X_{1}<1 / y\right)\right] y^{n a-1} d y \\
& =n\left[E\left(X_{1}^{-a}\right)\right]^{n-1} a \int_{0}^{\infty} P\left(X_{1}<1 / y\right) y^{a-1} d y=n\left[E\left(X_{1}^{-a}\right)\right]^{n}
\end{aligned}
$$

(

Proposition 4.8. Assume $\alpha_{0}=p_{0}=p_{1}=0$ and write $m=\min \{i \geq 2$ : $\left.p_{i}>0\right\}$. Let $0<a<\infty$ be a positive number. Then: (i) If $E\left(A^{-a}\right)<\infty$, then $E\left(Z^{-m a}\right)<\infty$; (ii) Let $t_{0}>0$ and let $l:\left[t_{0}, \infty\right) \rightarrow(0, \infty)$ be a function slowly varying at $\infty$ and bounded away from 0 and $\infty$ on every compact subset of $\left[t_{0}, \infty\right)$. If $E e^{-A t} \sim t^{-a} l(t)(t \rightarrow \infty)$, then

$$
E e^{-Z t} \sim p_{m} t^{-m a}\left[E\left(Z^{-a}\right)\right]^{m}[l(t)]^{m}, \quad t \rightarrow \infty .
$$

Proof. (i) Assume $E\left(A^{-a}\right)<\infty$. Then $P(A \leq x)=O\left(x^{a}\right)(x \rightarrow 0)$. So by Lemma 4.3 and Proposition 4.5, $P(Z \leq x)=O\left(x^{m a}\right)(x \rightarrow 0)$ and $E\left(Z^{-m b}\right)<\infty$ for all $0<b<a$. In particular, $E\left[(A Z)^{-a}\right]=E\left[A^{-a}\right] E\left[Z^{-a}\right]<\infty$. So by the equation (E) and Lemma 4.7,

$$
\begin{aligned}
E\left[Z^{-m a}\right] & =E\left[\left(A_{1} Z_{1}+\ldots+A_{N} Z_{N}\right)^{-m a}\right] \\
& \leq E\left[\left(A_{1} Z_{1}+\ldots+A_{m} Z_{m}\right)^{-m a}\right] \leq m\left(E\left[(A Z)^{-a}\right]\right)^{m}<\infty .
\end{aligned}
$$

(ii) Write $\phi(t)=E e^{-t Z}$ and $\phi_{A}(t)=E e^{-t A}$. For simplicity we can assume $t_{0}=0$; otherwise we consider $\tilde{l}$ instead of $l$, where $\tilde{l}(t)=1$ if $0 \leq t<t_{0}$ and $\tilde{l}(t)=l(t)$ if $t \geq t_{0}$.

Let $0<\epsilon<a$. So $E Z^{-(a+\epsilon)}<\infty$ by part (i). By Potter's theorem [10, p.25, Theorem 1.5.6(ii)], there exists $C_{1}=C_{1}(\epsilon)>0$ such that for all $x>0$ and $y>0$,

$$
l(y) / l(x) \leq C_{1} \max \left\{(y / x)^{\epsilon},(x / y)^{\epsilon}\right\} .
$$

Since $\phi_{A}(t) \sim t^{-a} l(t)$, we can suppose that there is some constant $C_{2}>0$ such that, for all $t>0, \phi_{A}(t) t^{a} / l(t) \leq C_{2}$. As $\phi_{A}(Z t) t^{a} / l(t) \rightarrow Z^{-a}(t \rightarrow \infty)$ and

$$
\begin{aligned}
\frac{\phi_{A}(Z t) t^{a}}{l(t)} & =\frac{\phi_{A}(Z t)(Z t)^{a}}{l(Z t)} \frac{l(Z t)}{l(t)} Z^{-a} \\
& \leq C_{2} C_{1} \max \left(Z^{\epsilon}, Z^{-\epsilon}\right) Z^{-a} \leq C_{2} C_{1}\left(Z^{-(a-\epsilon)}+Z^{-(a+\epsilon)}\right)
\end{aligned}
$$


by the dominated convergence theorem we obtain $\lim _{t \rightarrow \infty} \frac{E \phi_{A}(Z t) t^{a}}{l(t)}=E Z^{-a}$. So $E \phi(A t)=E \phi_{A}(Z t) \sim t^{-a} E\left(Z^{-a}\right) l(t)$. Therefore, by the equation (E'), as $t \rightarrow \infty$,

$$
\phi(t) \sim p_{m}[E \phi(A t)]^{m} \sim p_{m} t^{-a m}\left[E\left(Z^{-a}\right)\right]^{m}[l(t)]^{m} .
$$

Proposition 4.9. Assume $\alpha_{0}=p_{0}=0$ and $p_{1}>0$. Let $0<a<\infty$ be a positive number. Then: (i) If $p_{1} E\left(A^{-a}\right)<1$, then for all $0<b<a, E Z^{-b}<\infty$; if additionally $A \leq 1$ a.s., then $E Z^{-a}<\infty$. (ii) Conversely, if $E\left(Z^{-a}\right)<\infty$, then $p_{1} E\left(A^{-a}\right)<1$.

Proof. (i) If $p_{1} E\left(A^{-a}\right)<1$, then $E e^{-t Z}=O\left(t^{-a}\right)(t \rightarrow \infty)$ by Lemma 3.5. So for all $0<b<a, E Z^{-b}<\infty$. This gives the first conclusion. Now assume $P(A \leq 1)=1$. Then for all $0<b<a, E\left(A_{i} Z_{i}\right)^{-b}<\infty, E e^{-t\left(A_{1} Z_{1}+A_{2} Z_{2}\right)}=O\left(t^{-2 b}\right)$, so that $E\left[\left(A_{1} Z_{1}+A_{2} Z_{2}\right)^{-a}\right]<\infty$ by Lemma 4.3. Now for all $T>0$,

$$
\begin{aligned}
E Z^{-a} 1\{Z>T\} & =E\left(\sum_{i=1}^{N} A_{i} Z_{i}\right) 1\left\{\sum_{i=1}^{N} A_{i} Z_{i}>T\right\} \\
& \left.\leq E\left[\left(A_{1} Z_{1}\right)^{-a} 1\left\{A_{1} Z_{1}>T\right)\right\} 1\{N=1\}\right]+E\left[\left(A_{1} Z_{1}+A_{2} Z_{2}\right)^{-a}\right] P(N>1) .
\end{aligned}
$$

Because a. s. $\left.\left.A_{1} \leq 1,1\left\{A_{1} Z_{1}>T\right)\right\} \leq 1\left\{Z_{1}>T\right)\right\}$, it follows that

$$
\left(1-p_{1} E A^{-a}\right) E\left[Z^{-a} 1\{Z>T\}\right] \leq\left(1-p_{1}\right) E\left[\left(A_{1} Z_{1}+A_{2} Z_{2}\right)^{-a}\right] .
$$

Since $T>0$ is arbitrary, this gives $E Z^{-a} \leq\left(1-p_{1}\right) E\left[\left(A_{1} Z_{1}+A_{2} Z_{2}\right)^{-a}\right] /\left(1-p_{1} E A^{-a}\right)$.

(ii) Conversely, if $E\left(Z^{-a}\right)<\infty$, then by the equation (E),

$$
E\left[Z^{-a}\right]=E\left[\left(A_{1} Z_{1}+\ldots+A_{N} Z_{N}\right)^{-a}\right]>E\left[\left(A_{1} Z_{1}\right)^{-a} 1\{N=1\}\right]=p_{1} E\left[A^{-a}\right] E\left[Z^{-a}\right] .
$$

Therefore $p_{1} E\left[A^{-a}\right]<1$.

We now give a principle of reduction, which says that the case where $\alpha_{0}>0$ or $p_{0}>0$ can be reduced to the case where $\alpha_{0}=p_{0}=0$.

Proposition 4.10. Let $\bar{A}$ (resp. $\bar{Z}$ ) be a random variable whose distribution is that of $A$ (resp. Z) conditional on $A>0$ (resp. $Z>0$ ). Let $\bar{\phi}$ be the characteristic function of $\bar{Z}$ and put

$$
\bar{f}(t)=\frac{\tilde{f}(q+(1-q) t)-q}{1-q},
$$

where $q=P(Z=0)$ is the unique fixed point in $[0,1)$ of $\tilde{f}(t)=f\left(\alpha_{0}+\left(1-\alpha_{0}\right) t\right)$. Then the functional equation $\left(E^{\prime}\right)$ reduces to

$$
\bar{\phi}(t)=\bar{f}(E \bar{\phi}(\bar{A} t)), \quad t \in \mathbb{R},
$$

with $P(\bar{A}=0)=\bar{f}(0)=0$. Moreover, $\bar{f}^{\prime}(0)=\left(1-\alpha_{0}\right) f^{\prime}\left(\alpha_{0}+\left(1-\alpha_{0}\right) q\right)$, so that $\bar{f}^{\prime}(0)=0$ if and only if $\alpha_{0}=p_{0}=p_{1}=0$.

Proof. It suffices to remark that $E \phi(A t)=E \phi(A t) 1\{A>0\}+E \phi(A t) 1\{A=$ $0\}=\alpha_{0}+\left(1-\alpha_{0}\right) E \phi(\bar{A} t)$, that $\phi(t)=q+(1-q) \bar{\phi}(t)$, and that $f^{\prime}\left(\alpha_{0}+\left(1-\alpha_{0}\right) q\right)=$ $0 \Leftrightarrow f^{\prime}(0)=0$ and $\alpha_{0}+\left(1-\alpha_{0}\right) q=0$. 
For the Mandelbrot's equation ( $N$ is constant), the idea to consider the distribution of $A$ conditional on $A>0$ has been used by Barral [3]; for the Poincaré's equation (A is constant), the principle reduces to the famous transformation of Harris [20].

Proof of Theorem 4.1. Part (i) is a combination of Lemma 4.3, Proposition 4.5(i) and Lemma 4.6; part (ii) is a sequence of part (i) and Lemma 4.6; part (iii) comes from Propositions 4.5 and 4.8(i), together with Lemma 4.3; part (iv) follows from Proposition 4.8(ii) and a classical Tauberian theorem [10, Theorem 1.7.1].

Proof of Theorem 4.2. By the principle of reduction (Proposition 4.10), we can assume $p_{0}=\alpha_{0}=0$; the theorem is then Proposition 4.9.

It will be interesting to compare the left tail behavior with the right tail behavior; to this end let us state a theorem about the right tail behavior. The problem is called lattice if for some $h>0, \log A$ is almost surely an integer multiple of $h$ whenever $A>0$; otherwise, it is called non-lattice. The following result is a direct consequence of Theorems 1 and 2 of Liu [35]:

Proposition 4.11. Assume (1.6) and let $Z$ be a non-trivial and non-negative solution of $(E)$.

(i) For each fixed $p>1, E\left[Z^{p}\right]<\infty$ if and only if $E\left[N^{p}\right]<\infty$ and $E N E\left[A^{p}\right]<1$.

(ii) Suppose that for some $\chi>1, E N E\left[A^{\chi}\right]=1, E\left[A^{\chi} \log ^{+} A\right]<\infty$ and $E\left[N^{\chi}\right]<$ $\infty$. If the problem is non-lattice, then $\lim _{x \rightarrow \infty} x^{\chi} P(Z>x)$ exists and is strictly positive and finite; if the problem is lattice, then $0<$ $\liminf _{x \rightarrow+\infty} x^{\chi} P(Z>x) \leq \limsup _{x \rightarrow+\infty} x^{\chi} P(Z>x)<\infty$.

The result shows that if $P(A \leq 1)=1$, then the right tail of $Z$ behaves like that of $N$ : for all $p>1, E\left[Z^{p}\right]<\infty$ if and only if $E\left[N^{p}\right]<\infty$; if $P(A \leq 1)<1$, then there is a critical value $\chi>1$ determined by the equation $E N E\left[A^{\chi}\right]=1$ such that the right tail of $\mathrm{Z}$ behaves like $x^{-\chi}$ as $x \rightarrow \infty$. Theorems 4.1 and 4.2 tell us that there is a similar phenomenon for the left tail of $Z$ : if $\alpha_{0}+p_{0}+p_{1}=0$, then the left tail of $Z$ behaves like that of $A$; if $\alpha_{0}+p_{0}+p_{1}>0$ then there is a critical value $\lambda>0$ determined by $\tilde{p}_{1} E\left[A^{-\lambda} \mid A>0\right]=1$, such that the left tail of $Z$ behaves like $x^{\lambda}$ as $x \rightarrow 0$.

5. Exponential Tail Behavior of $\mu \in \mathbb{F}_{+}$. This section deals with the case where $P(Z \leq x)$ decays exponentially when $x \rightarrow 0+$. As usual, we always assume (1.3) and (1.4).

THEOREM 5.1. Suppose that

$$
m:=\operatorname{essinf} N>1 \text { and } a:=\operatorname{essinf} A>0,
$$

and put $\gamma=-\log m / \log a$. Then the following assertions hold:

(i) Assume (1.5) and that either $N$ or $A$ is not a. s. constant, and let $Z$ be a non-trivial and non-negative solution of $(E)$. Then $0<\gamma<1$ and, for all $\epsilon>0$, there are some constants $C_{1}>0$ and $C_{2}>0$ such that for all $x>0$ small enough,

$$
\exp \left\{-C_{1} x^{-\left(\frac{\gamma}{1-\gamma}+\epsilon\right)}\right\} \leq P(Z \leq x) \leq \exp \left\{-C_{2} x^{-\left(\frac{\gamma}{1-\gamma}\right)}\right\}
$$

moreover, the conclusion is also valid for $\epsilon=0$ if additionally $P\left(A=\|A\|_{-\infty}\right)>0$.

(ii) Assume (1.8) and let $Z$ be any non-trivial solution of (E). Then for some constant $K>0$ and all $t \in \mathbb{R}$ with $|t| \geq 1$,

$$
\left|E e^{i t Z}\right| \leq e^{-K|t|^{\gamma}}
$$


The theorem can be applied to the study of exact packing measures of some random Cantor- like sets [34]. In the context of Galton-Watson process (thus $A$ is constant), part (ii) is due to Harris (1948), and part (i) was deduced from a result of Harris (1948) by Bingham (1988), using Tauberian theorems. For the proof, we need the following proposition.

Proposition 5.2. Assume (1.5) and (5.1), and let $Z$ be a non-trivial and nonnegative solution of $(E)$. Then the following assertions hold:

(i) For some constant $K_{1}>0$ and all $t \geq 1, E e^{-t Z} \leq e^{-K_{1} t^{\gamma}}$.

(ii) For all $\epsilon>0$, there exists some constant $K_{2}>0$ such that for all $t \geq 1$, $E e^{-t Z} \geq e^{-K_{2} t^{\gamma+\epsilon}} ;$ the conclusion also holds for $\epsilon=0$ if $P(A=a)>0$.

Proof. We first remark that $a<1$ by (1.5). Write $\phi(t)=E e^{-t Z}$. By (E'), we have

$$
\phi(t) \leq[\phi(a t)]^{m} \text { for all } t>0 .
$$

So for $b=1 / a$ and $t>0, \phi(b t) \leq[\phi(t)]^{m}$ and, by iteration, for all $k=0,1, \ldots$, $\phi\left(b^{k}\right) \leq[\phi(1)]^{m^{k}}$. Since $m=a^{-\gamma}=b^{\gamma}$, it shows that, for all $k=0,1, \ldots$,

$$
-\log \phi\left(b^{k}\right) \geq K\left(b^{k}\right)^{\gamma}, \text { where } K=-\log \phi(1)>0 .
$$

For all $t \geq 1$, let $k \in\{0,1, \ldots\}$ be such that $b^{k} \leq x<b^{k+1}$. Then by the monotonicity of $\phi$,

$$
-\log \phi(t) \geq-\log \phi\left(b^{k}\right) \geq K\left(b^{k}\right)^{\gamma} \geq K a^{\gamma}\left(b^{k+1}\right)^{\gamma} \geq K a^{\gamma} t^{\gamma} .
$$

This ends the proof of part (i). We now come to the proof of part (ii). Let $\epsilon>0$ be such that $a+\epsilon<1$. Again by the equation (E'), we have

$$
\begin{aligned}
\phi(t) & \geq p_{m}(E[\phi(A t)])^{m} \geq p_{m}(E[\phi(A t) 1\{A \leq a+\epsilon\}])^{m} \\
& \left.\geq p_{m}(P\{A \leq a+\epsilon\})\right)^{m}[\phi((a+\epsilon) t)]^{m} .
\end{aligned}
$$

Therefore, for $\left.b=b_{\epsilon}:=1 /(a+\epsilon)>1, p=p_{\epsilon}:=p_{m}(P\{A \leq a+\epsilon\})\right)^{m} \in(0,1)$ and all $t>0, \phi(b t) \geq p[\phi(t)]^{m}$. Iterating, we obtain

$$
\phi\left(b^{k}\right) \geq[\phi(1)]^{m^{k}} p^{\sum_{1 \leq i \leq k} m^{i}}, \quad k=0,1, \ldots
$$

(where the empty sum is taken to be 0 ). It follows that for all $k=0,1, \ldots$,

$$
\begin{aligned}
-\log \phi\left(b^{k}\right) & \leq m^{k}\left[-\log \phi(1)+(-\log p) m^{-k} \sum_{1 \leq i \leq k} m^{i}\right] \\
& =m^{k}\left[-\log \phi(1)+(-\log p) m^{-k} m\left(m^{k}-1\right) /(m-1)\right] \leq\left(b^{k}\right)^{\gamma_{\epsilon}} K_{\epsilon}
\end{aligned}
$$

where $\gamma_{\epsilon}:=\log m / \log \frac{1}{a+\epsilon}$ and $K_{\epsilon}:=-\log \phi(1)+(-\log p) m /(m-1)$. As in the proof of part (i), together with the monotonicity of $\phi$, this implies that, for all $t \geq 1$,

$$
-\log \phi(t) \leq K_{\epsilon} a^{-\gamma_{\epsilon}} t^{\gamma_{\epsilon}} .
$$

As $\epsilon>0$ is arbitrary, this gives the first assertion in part (ii) of the theorem. If additionally $P(A=a)>0$, it is easily seen that the preceding argument also holds for $\epsilon=0$, giving the second assertion in part (ii). So the proof is finished. 
In the context of Mandelbrot's cascades (thus $N$ is constant), part (i) was established by Holley and Waymire [21]; in the context of Galton-Watson process (thus $A$ is constant), both parts are due to Harris [20].

Proof of Theorem 5.1. By (1.5) and the condition that either $N$ or $A$ is not a. s. constant, we know that $\gamma<1$. So part (i) follows from Tauberian theorems of exponential type [28, Theorem 3 (ii) \& (iii)]. Part (ii) can be shown in a similar way as in the proof of part (i) of Proposition 5.2 by considering $\bar{\phi}(t)=\sup _{|s| \geq t}\left|E e^{i s Z}\right|$ instead of $\phi(t)$.

The following results are consequences of Theorem 5.1, again by Tauberian theorems [30].

Corollary. Assume (1.5), (5.1) and that either $N$ or $A$ is not a. s. constant, and let $Z$ be a non-trivial and non-negative solution of (E). Then:

(i) For all $\epsilon>0$, there exist some constants $C_{i}>0(i=3,4)$ such that for all $k \geq 1, C_{3} k^{\frac{1-\gamma}{\gamma}-\epsilon} \leq\left\|Z^{-1}\right\|_{k} \leq C_{4} k^{\frac{1-\gamma}{\gamma}}$, where $\left\|Z^{-1}\right\|_{k}=\left(E\left[Z^{-k}\right]\right)^{1 / k}$. Consequently, $\lim _{k \rightarrow \infty} \log \left\|Z^{-1}\right\|_{k} / \log k=(1-\gamma) / \gamma$.

(ii) If $\gamma>1 / 2$, then for all $\epsilon>0$, there are some constants $C_{5}>0$ and $C_{6}>0$ such that, for all $t>0$ large enough, $C_{5} t^{\frac{\gamma}{2 \gamma-1}-\epsilon} \leq \log E e^{t Z^{-1}} \leq C_{6} t^{\frac{\gamma}{2 \gamma-1}}$ (so that $Z^{-1}$ has an entire characteristic function of order $\frac{\gamma}{2 \gamma-1}>1$ ).

(iii) $E \exp \left(Z^{-q}\right)<\infty$ if $q<\frac{\gamma}{1-\gamma}$, and $E \exp \left(Z^{-q}\right)=\infty$ if $q>\frac{\gamma}{1-\gamma}$.

(iv) If $P(A=a)>0$, then the conclusions in (i) and (ii) also hold for $\epsilon=0$, and the assertion (iii) can be improved to the following: for some but not all $r>0, E \exp \left(r Z^{-\gamma /(1-\gamma)}\right)<\infty$.

Again it is interesting to compare exponential left tail with exponential right tail. The corresponding results for the right tail are the following:

Proposition 5.3. Assume (1.6) and let $Z$ be a non-trivial and non-negative solution of $(E)$. Suppose that either $N$ or $A$ is not almost surely constant, and that $\bar{m}:=\operatorname{ess} \sup N<\infty$ and $\bar{a}:=\operatorname{ess} \sup A<1$. Write $\bar{\gamma}=-\log \bar{m} / \log \bar{a}$. Then $\bar{\gamma}>1$ and for any $\epsilon>0$, there exist some constants $C_{i}>0(1 \leq i \leq 6)$ such that the following assertions hold:

(i) For all $t \geq 1, C_{1} t^{\bar{\gamma}+\epsilon} \leq \log E e^{t Z} \leq C_{2} t^{\bar{\gamma}}$ (therefore $Z$ has an entire characteristic function of regular growth with order $\bar{\gamma})$.

(ii) For all $x>0$ large enough, $\exp \left\{-C_{3} x^{\left(\frac{\bar{\gamma}}{\bar{\gamma}-1}+\epsilon\right)}\right\} \leq P(Z>x) \leq$ $\exp \left\{-C_{4} x^{\left(\frac{\bar{\gamma}}{\bar{\gamma}-1}\right)}\right\}$.

(iii) For all $k \geq 1, C_{5} k^{\frac{\bar{\gamma}-1}{\bar{\gamma}}-\epsilon} \leq\|Z\|_{k} \leq C_{6} k^{\frac{\bar{\gamma}-1}{\bar{\gamma}}}$, where $\|Z\|_{k}=\left(E\left[Z^{k}\right]\right)^{1 / k}$. Consequently, $\lim _{k \rightarrow \infty} \log \|Z\|_{k} / \log k=(\bar{\gamma}-1) / \bar{\gamma}$.

(iv) $E \exp \left(Z^{q}\right)<\infty$ if $q<\frac{\bar{\gamma}}{\bar{\gamma}-1}$, and $E \exp \left(Z^{q}\right)=\infty$ if $q>\frac{\bar{\gamma}}{\bar{\gamma}-1}$.

(v) If additionally $P(Z=\bar{a})>0$, then the conclusions in (i), (ii) and (iii) also hold for $\epsilon=0$, and the conclusions in (iv) can be improved to the following: for some but not all $r>0, E \exp \left(r Z^{\bar{\gamma} /(\bar{\gamma}-1)}\right)<\infty$.

Part (i) can be obtained in a similar way as in the proof of Proposition 5.2 by considering $E e^{t Z}$ (which can be shown finite) instead of $E e^{-t Z}, t>0$; other parts are consequences of Part (i) by Tauberian Theorems [30]. In fact, by [30, Corollary 1.2], the condition $P(Z=\bar{a})>0$ in (v) can be relaxed to the following: for some constants $c>0, a \geq 0$ and all $x>0$ small enough,

$$
P(A / \bar{a}>1-x) \geq c x^{a} .
$$


Because the proof in [30] is not easy, the new approach seems to be of interest.

6. Martingales in Self-similar Cascades. Fix an integer $r \geq 2$, and write $T=\cup_{n=0}^{\infty}\{1, \ldots, r\}^{n}$ for the set of finite sequences of integers between 1 and $r$. Let $\left\{A_{u}\right\}_{u \in T}$ be a family of independent random variables, each distributed as $A \geq 0$ with $E A=1 / r$. Put

$$
Y_{n}=\sum_{u \in T,|u|=n} X_{u}, \text { where } X_{u}=A_{u_{1} \ldots A_{u_{1} \ldots u_{n}}} \text { if } u=u_{1} \ldots u_{n}, n \geq 1
$$

( $|u|$ being the length of the sequence $u$ ). Then $\left\{Y_{n}\right\}$ is a non-negative martingale (associated with the natural $\sigma$-algebra), which converges (when $n \rightarrow \infty$ ) almost surely to a random variable $Y_{\infty} \geq 0$ satisfying the equation (E) with $N=r$ (we shall always assume this in this section) and $E Y_{\infty} \leq 1$. The study of the limit variable $Y_{\infty}$ and the equation (E) plays an essential role in the investigation of the Mandelbrot's cascade $[39,40,27,19]$. It has been known [27] that $E Y_{\infty}=1$ if

$$
E A \log A<0,
$$

and $Y_{\infty}=0$ a.s. otherwise. It has also been known that [35] if

$$
E A \log A=0 \text { and } E A^{1+\epsilon}<\infty \text { for some } \epsilon>0
$$

then

$$
Y_{n}^{*}=\sum_{u \in T,|u|=n} X_{u} \log \frac{1}{X_{u}}
$$

is a martingale, and converges to a a solution $Y_{\infty}^{*} \geq 0$ of $(\mathrm{E})$ with $\lim _{t \rightarrow 0+} \frac{1-E e^{-t Y_{\infty}^{*}}}{t|\log t|}=$ 1, so that $E Y_{\infty}^{*}=\infty$. Since both $Y_{\infty}$ and $Y_{\infty}^{*}$ are non-negative solutions of $(E)$, our theorems can be applied to get new results on them; as examples, let us deduce some of these results in the following. Remark that by our notations, we have: (i) $\tilde{p}_{1}=\left(1-\alpha_{0}\right) r q /\left[\alpha_{0}+\left(1-\alpha_{0}\right) q\right]$ where $\alpha_{0}=P(A=0), q=P(Z=0)$ is the unique fixed point in $[0,1)$ of $\tilde{f}(t)=\left[\alpha_{0}+\left(1-\alpha_{0}\right) t\right]^{r}$; (ii) $m=r$ if $\alpha_{0}=0$ and $m=1$ if $\alpha_{0}>0$.

\section{THEOREM 6.1.}

We assume (6.2) if $Y_{\infty}$ is concerned and (6.3) if $Y_{\infty}^{*}$ is concerned.

(i) Let $\phi_{\infty}$ be the characteristic function of $Y_{\infty}$, and let $\phi_{\infty}^{\prime}$ be its derivative. Then $\phi_{\infty}^{\prime}(t)=O\left(|t|^{-1}\right) \quad(|t| \rightarrow \infty)$ and $\int_{\mathbb{R}}\left|\phi_{\infty}^{\prime}(t)\right| d t<\infty$, so that the distribution of $Y_{\infty}$ has a continuous density function on $(0, \infty)$, given by $x \mapsto$ $\frac{1}{2 \pi i x} \int_{\mathbb{R}} \phi_{\infty}^{\prime}(t) e^{-i x t} d t$. If for some $a \geq 0$, either (a) $\alpha_{0}=0$ and $E A^{-a}<\infty$ or (b) $\alpha_{0}>0$ and $\tilde{p}_{1} E\left[A^{-a} \mid A>0\right]<1$, then $\phi^{\prime}(t)=O\left(|t|^{-(m a+1)}\right)(|t| \rightarrow \infty)$ and $\int_{\mathbb{R}}\left|\phi_{\infty}^{\prime}(t)\right||t|^{m a} d t<\infty$, so that the density function given above is of class $C^{[m a]}$.

(ii) If for some $a>0$ either (a) or (b) of (i) holds, then $E\left[e^{i t Y_{\infty}^{*}} \mid Y_{\infty}^{*}>0\right]=$ $O\left(|t|^{-m a}\right) \quad(|t| \rightarrow \infty)$ and $\int_{\mathbb{R}}\left|E\left[e^{i t Y_{\infty}^{*}} \mid Y_{\infty}^{*}>0\right]\right||t|^{m a-1} d t<\infty$, so that the distribution of $Y_{\infty}^{*}$ has a square integrable density function on $(0, \infty)$ if $a>$ $1 /(2 m)$, and has a density function of class $C^{[m a]-1}$ if $a \geq 1 / m$.

Notice that the assertion (ii) also holds for $Y_{\infty}$ instead of $Y_{\infty}^{*}$. The results follow from Theorems 3.2, 3.4, 3.5 and the principle of reduction (Proposition 4.10). The 
fact that $Y_{\infty}$ has always a continuous density function on $(0, \infty)$ is also a consequence of a result of Biggins and Grey [6].

THEOREM 6.2. Assume (6.2) if $Y_{\infty}$ is concerned and (6.3) if $Y_{\infty}^{*}$ is concerned, and let $Z$ be either $Y_{\infty}$ or $Y_{\infty}^{*}$. If $\alpha_{0}=0$, then for each fixed $0<a<\infty$, the following assertions hold:

(i) As $x \rightarrow 0, P(A \leq x)=O\left(x^{a}\right)$ if and only if $P(Z \leq x)=O\left(x^{r a}\right)$.

(ii) If for some constants $C_{1}>0, C_{2}>0$ and all $x>0$ small enough, $C_{1} x^{a} \leq$ $P(A \leq x) \leq C_{2} x^{a}$, then there are some constants $C_{3}>0, C_{4}>0$ such that, for all $x>0$ small enough,

$$
C_{3} x^{r a} \leq P(Z \leq x) \leq C_{4} x^{r a} .
$$

(iii) If $E\left(A^{-a}\right)<\infty$, then $E\left[Z^{-r a}\right]<\infty$; conversely, if $E\left[Z^{-r a}\right]<\infty$, then $E\left(A^{-b}\right)<\infty$ for all $0<b<a$.

(iv) Let $\delta>0$ and let $l:(0, \delta] \rightarrow(0, \infty)$ be a function slowly varying at 0 and bounded away from 0 and $\infty$ on each compact subset of $(0, \delta]$. If $P(A \leq x) \sim$ $x^{a} l(x)(x \rightarrow 0)$, then

$$
P(Z \leq x) \sim x^{r a}\left[E\left(Z^{-a}\right)\right]^{r}[l(x)]^{r}(x \rightarrow 0) .
$$

The results follow from Theorem 4.1. By the remark following Theorem 4.1, the converse in (iii) cannot be improved to " $E Z^{-r a}<\infty \Rightarrow E A^{-a}<\infty$ ". In (i), the "only if" part was shown independently by Kahane [26], Molchan [41] and Barral $[3,4]$, and the "if" part can be deduced from the proof of Theorem II.A' of Barral [4]; in (iii), the first conclusion was due to Molchan [41, Theorem 4] and the second due to Barral [3, 4]; other results are new.

Theorem 6.3. Assume (6.2) if $Y_{\infty}$ is concerned, and (6.3) if $Y_{\infty}^{*}$ is concerned. Let $Z$ be either $Y_{\infty}$ or $Y_{\infty}^{*}$. If $\alpha_{0}>0$, then $0<\tilde{p}_{1}<1$, and for each fixed $0<a<\infty$, the following assertions hold:

(i) If $\tilde{p}_{1} E\left[A^{-a} \mid A>0\right]<1$, then $E\left[Z^{-b} \mid Z>0\right]<\infty$ for all $0<b<a$; conversely, if $E\left[Z^{-a} \mid Z>0\right]<\infty$, then $\tilde{p}_{1} E\left[A^{-a} \mid A>0\right]<1$

(ii) In the case where $A \leq 1$ almost surely, $\tilde{p}_{1} E\left[A^{-a} \mid A>0\right]<1$ if and only if $E\left[Z^{-a} \mid Z>0\right]<\infty$

The results follow from Theorem 4.2. Barral [3, 4] showed that when $\alpha_{0}>0$, $E\left[Y_{\infty}^{-b} \mid Y_{\infty}>0\right]=\infty$ if $b$ is sufficiently large. Our result gives explicitly the critical value for existence of moments of negative orders:

Corollary. Under the hypotheses of Theorem 6.3 , If $\tilde{p}_{1} E\left[A^{-\lambda} \mid A>0\right]=1$ for some $\lambda>0$, then

$$
E\left[Z^{-b} \mid Z>0\right] \begin{cases}<\infty & \text { when } b \in(0, \lambda), \text { and } \\ =\infty & \text { when } b \in[\lambda, \infty) .\end{cases}
$$

Notice that this result is useful in the study of multi-fractal structure of Mandelbrot's measures [3, 4, 41].

7. Applications to age-dependent processes . An age-dependent branching process - the Bellman-Harris process - can be described as follows. A particle existing at time 0 is assumed to have a life-length, $L$, with values in $[0, \infty)$ and probability distribution $G(x)=P(L \leq x)$. At the end of its life, it is transformed into $N$ particles according as a probability law $\left\{p_{n}: n \geq 0\right\}$ on $\mathbb{N}=\{0,1, \ldots\}: P(N=$ 
$n)=p_{n}, \sum_{n \in \mathbb{N}} p_{n}=1$. These new particles are taken to have the same life-length distribution and transformation probabilities as the original one. We assume that the life-length distribution and the transformation probabilities for each particle is independent of its time of birth and the number of other particles existing at the time. Let $Z(t)$ be the number of particles existing at time $t \geq 0$. As usual, we assume

$$
p_{0}+p_{1}<1,1<E N<\infty \text { and } P(L=0)<1 \text {. }
$$

It is well-known that the limit

$$
W=\lim _{t \rightarrow \infty} Z(t) / E Z(t)
$$

exists a.s., and its Laplace transform $\phi(s)=E e^{-s W}$ satisfies the functional equation

$$
\phi(s)=\int_{0}^{\infty} f\left(\phi\left(s e^{-\beta x}\right)\right) d G(x), \quad s>0,
$$

where $f(x)=\sum_{n=0}^{\infty} p_{n} x^{n}$ is the probability generating function of $N$, and $\beta$ is the Malthus parameter defined by $(E N) \int_{0}^{\infty} e^{-\beta x} d G(x)=1$. Notice that this equation also reads

$$
\phi(t)=E f(\phi(A t))
$$

with $A=e^{-\beta L}$ (so that $E N E A=1$ ); this corresponds to the equation (E) with all the coefficients $A_{i}$ replaced by $A$. It is known that $E W=1$ if $E N \log ^{+} N<\infty$, and $W=0$ almost surely otherwise; when $E N \log ^{+} N=\infty$, there are positive constants $\{C(t)\}$ such that

$$
\lim _{t \rightarrow \infty} Z(t) / C(t)=W^{\prime} \quad \text { almost surely }
$$

for some non-degenerate random variable $W^{\prime}$ whose Laplace transform $\phi$ satisfies (7.4)(Cf. [13] or [43]). The equations (E') and (7.4) are closely related. In fact, if $\phi$ is a solution of (7.4), then $\phi_{1}=f(\phi(t))$ is a solution of (E'); conversely, if $\phi_{1}$ is a solution of (7.4), then $\phi(t)=E \phi_{1}(A t)$ is a solution of (E). So properties of solutions of $(E)$ can be transfered to solutions of (7.4). Let us give an example to explain this.

ThEOREM 7.1. Assume $p_{0}=p_{1}=0$ and let $Z=W$ if $E N \log N<\infty$ and $Z=W^{\prime}$ if $E N \log N=\infty$. Then for each fixed $0<a<\infty$, the following assertions hold:

(i) As $x \rightarrow 0, P(A \leq x)=O\left(x^{a}\right)$ if and only if $P(Z \leq x)=O\left(x^{a}\right)$.

(ii) Let $\delta>0$ and let $l:(0, \delta] \rightarrow(0, \infty)$ be a function slowly varying at 0 and bounded away from 0 and $\infty$ on each compact subset of $(0, \delta]$. If $P(A \leq x) \sim$ $x^{a} l(x)(x \rightarrow 0)$, then

$$
P(Z \leq x) \sim c x^{a} l(x)(x \rightarrow 0),
$$

where $0<c=E\left(Z_{1}+\ldots+Z_{N}\right)^{-a}<\infty, Z_{k}$ being independent copies of $Z$ and independent of $N$.

Proof. Write $m=\min \left\{i \geq 2: p_{i}>0\right\}$ and let $\phi$ be the Laplace transform of $Z$. Since $Z^{\prime}=Z_{1}+\ldots+Z_{N}$ is a solution of (E) with Laplace transform $f(\phi(t)) \sim$ $p_{m}(\phi(t))^{m}$ as $t \rightarrow \infty$, the results follow from Theorem 4.1 and Tauberian theorems.

Part (i) was established in [33]; part (ii) is new. Some other known results on $W$ and $W^{\prime}$ can also be obtained from our results on the equation (E). The equation (7.4) can also be considered for any given random variable $A \geq 0$ [33]. 
8. Applications to branching random walks. An i.i.d. branching random walk can be described as follows. Let $\mathbb{N}^{*}=\{1,2, \cdots\}$ be the set of positive integers and let $U=\{\emptyset\} \cup \cup_{k=1}^{\infty}\left(\mathbb{N}^{*}\right)^{k}$ be the set of all finite sequences including the null sequence $\emptyset$. Let $(\Omega, \mathbb{F}, P)$ be a probability space and let $\left\{N_{u}: u \in \mathbb{U}\right\}$ and $\left\{L_{u}: u \in\right.$ $\mathbf{U}\}$ be two independent families of independent random variables defined on $(\Omega, \mathbb{F}, P)$, the $N_{u}^{\prime} s$ are distributed as $N=N_{\emptyset}$ and with values on $\{0,1, \ldots\}$, and the $L_{u}^{\prime} s$ are distributed as $L=L_{\emptyset}$ ) and with values on $\mathbb{R}=(-\infty, \infty)$. ( $L$ is now not necessarily non-negative. ) Let $\mathbb{T}=\mathbb{T}(\omega)$ be the Galton -Watson tree with defining elements $\left\{N_{u}\right\}$ - we have $\emptyset \in \mathbb{T}$ and, if $u \in \mathbb{T}$ and $i \in \mathbb{N}$, then $u i \in \mathbb{T}$ if and only if $1 \leq i \leq N_{u}$ -. The initial particle $\emptyset \in \mathbb{T}$ is placed at $S_{\emptyset}=0$ of the real line $\mathbb{R}=(-\infty, \infty)$. It gives birth to $N_{\emptyset}$ new particles $i\left(1 \leq i \leq N_{\emptyset}\right)$ with displacements $L_{i}, 1 \leq i \leq N$. In general, if $u=u_{1} \ldots u_{n} \in \mathbb{T}$ is a particle in $\mathrm{n}$-th generation $(u=\emptyset$ if $n=0)$, then its position is given by

$$
S_{u}=L_{u_{1}}+\ldots+L_{u_{1} \ldots u_{n}}
$$

Assume (7.1) and let $\beta \in \mathbb{R}$ be such that $m(\beta):=E N E e^{-\beta L}<\infty$. Then the sequence

$$
Y_{n}:=m(\beta)^{-n} \sum_{u=u_{1} \ldots u_{n} \in T} e^{-\beta S_{u}}, \quad n \geq 1
$$

is a martingale, so that the limit

$$
Z:=\lim _{n \rightarrow \infty} Y_{n}
$$

exists almost surely. By considering the sub-trees beginning at $i \in\{1, \ldots, N\}$, we see easily that $Z$ satisfies the distributional equation (E) with $A_{i}=e^{-\alpha L_{i}} / m(\alpha)$. In the following, we always write $A=e^{-\beta L} / m(\beta)$, so $E N E A=1$.

Let $Z$ be the random variable defined by (8.1). It is known (see for example [31]) that the distribution of $Z$ is the unique non-negative solution of $(\mathrm{E})$ with mean 1 if

$$
E N \log ^{+} N<\infty \text { and } E A \log ^{+} A<\infty \text { with } E A \log A<0
$$

and $Z=0$ a. s. otherwise. In the case where (8.2) fails, (E) has no non-negative and non-trivial solution with finite mean, but it does have non-negative and non-trivial solutions with infinite mean by Theorem 1.1. In fact, if

$$
E N \log ^{+} N=\infty \text { and } E A \log ^{+} A<\infty \text { with } E A \log A<0
$$

then there is a sequence of constants $\left\{c_{n}\right\}$ such that $Y_{n} / c_{n}$ converges in probability to a solution $Z^{\prime} \geq 0$ of $(E)$ with $E Z^{\prime}=\infty[7]$; if

$$
E\left(N^{1+\delta}\right)+E\left(A^{1+\delta}\right)<\infty \text { for some } \delta>0 \text { and } E A \log A=0,
$$

then

$$
Y_{n}^{*}:=\sum_{u=u_{1} \ldots u_{n} \in T(\omega)} X_{u} \log \frac{1}{X_{u}}, \text { where } X_{u}=m(\alpha)^{-n} e^{-\alpha S_{u}}
$$

is a martingale, and converges to a solution $Z^{*} \geq 0$ of $(\mathrm{E})$ with $\lim _{t \rightarrow 0}(1-$ $\left.E e^{-t Z^{*}}\right) /|t \log t|=1$, so that $E Z^{*}=\infty[35]$. 
Since $Z, Z^{\prime}$ and $Z^{*}$ are all solutions of $(\mathrm{E})$ with $\alpha_{0}=P(A=0)=0$, our Theorems $3.2,3.4,3.5,4.1,4.2,5.1$ show asymptotic properties of their distributional functions or characteristic functions, and prove the existence of a density function and its regularity under simple moment conditions. To my knowledge all these results are new, except for the absolute continuity of $Z$ (defined by (8.1)) which was shown by Biggins and Grey [6].

Acknowledgment. The author is very grateful to Yves Guivarc'h for helpful discussions, and to the referee for valuable comments and remarks. He also thanks Dimitri Pétritis for having corrected some errors in English.

\section{REFERENCES}

[1] AthreyA, K.B., On the supercritical one dimensional age dependent branching processes, Ann. Math. Statistics, 40 (1969), pp. 743-763.

[2] Athreya, K.B, On the absolute continuity of the limit random variable in the supercritical Galton-Watson branching process, Proc. Amer. Math. Soc., 30 (1971), pp. 563-565.

[3] Barral, J., Continuité, Moments d'ordres négatifs et Analyse Multifractale des cascades multiplicatives de Mandelbrot, Thèse, Univ. Paris-Sud, Orsay, (1997).

[4] Barral, J., Continuté, Moments d'ordres négatifs et Analyse Multifractale des cascades multiplicatives de Mandelbrot, prepublication (1999), pp. 97 22, Univ. Paris-sud, Orsay. See also: Moments, continuité, et analyse multifractale des cascades multiplicatives de Mandelbrot. Prob. Th. Rel. Fields, 113 (1999), pp. 535-569.

[5] Ben NASR, F., Mesures aléatoires de Mandelbrot associées à des substitutions, CRAS, Sér. I, 304 (1987), pp. 255-258.

[6] Biggins, J.D. AND GREY D.R., Continuity of limit random variables in the branching random walk, J. Appl. Prob., 16 (1979), pp. 740-749.

[7] Biggins, J.D. AND KyPrianou, A.E., Seneta-Heyde norming in the branching random walk, Ann. Prob., 25 (1997), pp. 337-360.

[8] Bingham, N.H., On the limit of a supercritical branching process, J. Appl. Prob., 25A (1988), pp. 215-228.

[9] Bingham, N.H. AND Doney, R.A., Asymptotic properties of supercritical branching processes I: The Galton-Watson process, Adv. Appl. Prob., 6 (1974), pp. 711-731.

[10] Bingham, N.H., Goldie, C.M. and Teugels, J.L., Regular variation, Cambridge: Cambridge University Press (1987).

[11] Chauvin, B. And Rouault, A., Boltzmann-Gibbs weights in the branching random walk, IMA Congress on Branching Processes $n^{\circ} 84$. Lecture Notes in Maths, (1996).

[12] Collet, P. AND Koukiou, F., Large deviations for multiplicative chaos, Comm. Math. Phys., 147 (1992), pp. 329-342.

[13] CoHN, H., Norming constants for the finite mean supercritical Bellman-Harris process, Z. Wahrsch., 61 (1982), pp. 189-205.

[14] Dubuc, S., La densité de la loi-limite d'un processus en cascade expansif, Z. Wahrsch. verw. Gebeite, 19 (1971), pp. 281-290.

[15] DurRett, R. AND LIGGetT, T., Fixed points of the smoothing transformation, Z. Wahrsch. verw. Gebeite, 64 (1983), pp. 275-301.

[16] ERDös, P., On the smoothness properties of a family of Bernoulli convolutions, Amer. J. Math., 62 (1940), pp. 180-186.

[17] Feller, W., An introduction to probability theory and its applications, vol.II, 2nd. ed (1970), John Wiley \& Sons, New York.

[18] GRINTSEVICHYUS, A.K., On the continuity of a sum of dependent variables connected with independent walks on lines, Theory Prob. Appl., 19 (1974), pp. 163-168.

[19] Guivarc'H, Y., Sur une extension de la notion de loi semi-stable, Ann. IHP, 26 (1990), pp. 261-285.

[20] Harris, T.E., Branching processes, Ann. Math. Stat., 19 (1948), pp. 474-494.

[21] Holley, R. AND WAYMire, E.C., Multifractal dimensions and scaling exponents for strongly bounded cascades, Ann. Appl. Prob., 2 (1992), pp. 819-845.

[22] HU, T.Y. AND LAU, K.S., The sum of Rademacher functions and Hausdorff dimension, Math. Proc. Camb. Phil. Soc., 108 (1990), pp. 97-103.

[23] HU, T.Y. AND LAU, K.S., personal communication, (1999). 
[24] Kahane, J.P., Sur la distribution de certaines sries aleatoires, Bull. Soc. Math. France, Memoire, 25 (1971), pp. 119-122.

[25] Kahane, J.P., Multiplications aléatoires et dimension de Hausdorff, Ann. IHP, Sup. au no. 2, vol. 23 (1987), pp. 289-296.

[26] Kahane, J.-P., Produit de poids aléatoires indépendants et applications. In Fractal geometry and analysis (eds. J. Bélaire and S. Dubuc), Kluwer Acad. Publ. 1991, pp. 277-324.

[27] Kahane, J.P. AND Peyrière, Sur certaines martingales de Benoit Mandelbrot, Adv. Math., 22 (1976), pp. 131-145.

[28] Kasahara, Y., Tauberian theorems of exponential type, J. Math. Kyoto Univ. (JMK YAZ), 18-2 (1978), pp. 209-219.

[29] Kesten, H. AND Stigum, B.P., A limit theorem for multidimensional Galton-Watson processes, Ann. Math. Statist., 37 (1966), pp. 1211-1223.

[30] LIU, Q., The growth of an entire characteristic function and the tail probabilities of the limit of a tree martingale. In Trees. Progress in Probability, vol.40 (1996), pp. 51-80, Birkhöuser: Verlag Basel. Eds.: B.Chauvin, S.Cohen, A.Rouault.

[31] LIU, Q., Sur une équation fonctionnelle et ses applications : une extension du théorème de Kesten-Stigum concernant des processus de branchement, Adv. Appl. Prob., 29 (1997), pp. 353-373.

[32] LIU, Q., Fixed points of a generalized smoothing transformation and applications to branching random walks, Adv. Appl. Prob., 30 (1998), pp. 85-112.

[33] LIU, Q., Asymptotic properties of supercritical age-dependent branching processes and homogeneous branching random walks, Stoch. Proc. Appl., 82 (1999), pp. 61-87.

[34] LIU, Q., Exact packing measures of random fractals, Preprint, Univ. Rennes 1, (1999).

[35] LIU, Q., On generalized multiplicative cascades, Stoch. Proc. Appl., 86 (2000), pp. 263-286.

[36] LIU, Q., Asymptotic properties and absolute continuity of laws stable by random weighted mean, Stoch. Proc. Appl., 95 (2001), pp. 83-107.

[37] Liu,Q. AND Rouault, A., On two measures defined on the boundary of a branching tree. In "Classical and modern branching processes", ed. K.B. Athreya and P. Jagers. IMA Volumes in Mathematics and its applications, vol. 84 (1996), pp. 187-202. Springer-Verlag.

[38] Liu,Q. AND RouAult, A., Limit theorems for Mandelbrot's multiplicative cascades, Ann. Appl. Prob., 10 (2000), pp. 218-239.

[39] MANDELbRot, B., Multiplications aléatoires et distributions invariantes par moyenne pondérée aléatoire, CRAS Paris, 278 (1974), pp. 325-346 et 355-358.

[40] MANDELBRot, B., Intermittent turbulence in self-similar cascades: divergence of high moments and dimension of the carrier, J.Fluid Mech., 62 (1974), pp. 331-333.

[41] Molchan, G.M., Scaling exponents and Multifractal Dimensions for independent random cascades, Comm. Math. Phys., 179 (1996), pp. 681-702.

[42] Rösler, U., A fixed point theorem for distributions, Stochastic processes and their applications, 42 (1992), pp. 195-214.

[43] Schum H.J., Seneta constants for the supercritical Bellman-Harris process, Adv. Appl. Prob., 14 (1982), pp. 732-751.

[44] Seneta, E., Functional equations and the Galton-Watson process, Adv. Appl. Prob., 1 (1969), pp. $1-42$.

[45] SolomYaK, B., On the random series $\sum \pm \lambda^{n}$ (an Erdös problem), Ann. Math., 142 (1995), pp. 611-625.

[46] Waymire, E.C. AND Williams, S.C., Multiplicative cascades : dimension spectra and dependence, J. of Fourier Analysis and Appl., Kahane Special Issue (1995), pp. 589-609. 